\title{
The conformal current algebra on supergroups with applications to the spectrum and integrability
}

\author{
Raphael Benichou ${ }^{a}$ and Jan Troost ${ }^{b}$ \\ ${ }^{a}$ Theoretische Natuurkunde, Vrije Universiteit Brussel, \\ Pleinlaan 2, B-1050 Brussels, Belgium \\ ${ }^{b}$ Laboratoire de Physique Théorique, Unité Mixte du CRNS et de l'École Normale Supérieure, \\ Associée à l'Université Pierre et Marie Curie 6, \\ UMR 8549 École Normale Supérieure, \\ 24 Rue Lhomond Paris 75005, France \\ E-mail: raphael.benichou@vub.ac.be, troost@lpt.ens.fr
}

Abstract: We compute the algebra of left and right currents for a principal chiral model with arbitrary Wess-Zumino term on supergroups with zero Killing form. We define primary fields for the current algebra that match the affine primaries at the WessZumino-Witten points. The Maurer-Cartan equation together with current conservation tightly constrain the current-current and current-primary operator product expansions. The Hilbert space of the theory is generated by acting with the currents on primary fields. We compute the conformal dimensions of a subset of these states in the large radius limit. The current algebra is shown to be consistent with the quantum integrability of these models to several orders in perturbation theory.

Keywords: Conformal Field Models in String Theory, Conformal and W Symmetry, Integrable Field Theories

ArXiv EPRINT: 1002.3712 


\section{Contents}

1 Introduction $\quad 2$

2 The conformal current algebra 3

3 The left-right current algebra $\quad 6$

$\begin{array}{ll}3.1 \text { The primary adjoint operator } & 6\end{array}$

3.2 The left current - right current OPEs 8

4 The primaries $\quad 10$

5 A recursive bootstrap for the elementary operator algebra 12

6 Composite operators and their conformal dimension $\quad 14$

$\begin{array}{llr}7 & \text { The classical and quantum integrability } & 18\end{array}$

8 Conclusions $\quad 19$

$\begin{array}{ll}\text { A Operator products involving composite operators } & 20\end{array}$

B The semi-classical behavior of the OPE coefficients 22

$\begin{array}{ll}\text { C Consistency of perturbation theory } & 25\end{array}$

$\begin{array}{ll}\text { D Conformal current algebra: precisions } & 27\end{array}$

$\begin{array}{ll}\text { D.1 The current algebra at order } f^{2} & 27\end{array}$

D.2 The Virasoro algebra from the current algebra 31

D.3 Currents as a primary fields of dimension one revisited 32

D.4 The associativity of the current algebra 34

$\begin{array}{ll}\text { D.5 The holomorphy of the stress-tensor } & 37\end{array}$

$\begin{array}{lll}\text { E Details on primary operators } & \mathbf{3 8}\end{array}$

E.1 Behavior of current primaries under perturbation of the kinetic term 38

E.2 Current-primary OPE at order $f^{2} \quad 40$

E.3 Stress-tensor-primary OPE at order $f^{2} \quad 41$

$\begin{array}{lll}\text { F The mode expansion on the cylinder } & 43\end{array}$

G Classical integrability $\quad 44$ 


\section{Introduction}

Principal chiral models with Wess-Zumino term on supergroups (and their cosets) arise in many contexts including string theory on Anti-de Sitter backgrounds with RamondRamond fluxes, the integer quantum hall effect, quenched disorder systems, polymers, as well as other domains in physics. When the supergroup has zero Killing form, the model is perturbatively conformal [1-3]. Thus, these models provide us with a two-parameter family of two-dimensional conformal field theories with supergroup symmetry. They exhibit a current algebra which is conformal and non-chiral [4]. Since these models fall into a class which exhibits integrability at least classically and most likely quantum mechanically, these two-dimensional conformal field theories may allow for an exact determination of their spectrum.

Steps towards solving these models were made using various techniques. For particular supergroups the Wess-Zumino-Witten points are well-understood [5-7]. The bulk spectrum was computed at some special points of the moduli space in [8]. The spectrum for states living on particular boundaries can be obtained at any point of the moduli space [911]. Methods to compute a subset of correlation functions were recently proposed in [12]. Despite these successes, the determination of the full bulk spectrum of the conformal field theories on supergroups remains an open problem.

A strong motivation for determining the spectrum of these models, and their cosets, is the prospect of solving string theory in AdS space-times in conformal gauge, which via holography [13] may lead to a neater formulation of the solution of gauge theories at large $N$ [14]. Our attitude in attacking this problem is to first attempt to solve for the spectrum in conformal gauge on supergroups (relevant to $A d S_{3}$ string theory for instance), and then for the spectrum on supercosets (relevant to $A d S_{5}$ string theory for example).

In this paper, we take a further step in our understanding of the symmetry, the integrability and the Hilbert space and spectrum of these models. In section 2, we review the conformal current algebra [4] obeyed by the conserved current associated to the left action of the supergroup on itself. We will determine further terms at order zero in the current algebra. In section 3 we compute the interplay between the left and the right conformal current algebra, as well as with the adjoint primary operator. In section 4 we define the primary fields for the current algebra. These fields correspond to the affine primaries at the Wess-Zumino-Witten points. We show that current primaries are also Virasoro primaries and compute their conformal dimension at large radius. In section 5 we explain how to compute the current-current and current-primary OPEs order by order in perturbation theory by demanding consistency with current conservation and the Maurer-Cartan equation. In section 6 we compute conformal dimensions of operators that are composites of a current and a primary to first order in semi-classical perturbation theory. We argue that the Hilbert space is generated by composites of currents and primary fields and show how to compute the conformal dimension of such operators in semi-classical perturbation theory. In section 7 we comment on the classical and quantum integrability of the model, and its consistency with the conformal current algebra. We conclude in section 8 .

We have gathered many technical details in the appendices. In appendix A we give a 
prescription to compute OPEs involving composite operators. In appendix B we compute the behavior at large radius for the coefficients appearing in the current-current and currentprimary OPEs. In appendix $\mathrm{C}$ we prove the consistency of the perturbative algorithm used to compute the current-current and current-primary OPEs. Appendix D contains further consistency checks of the current algebra as well as details of the computation of the current algebra. In appendix $\mathrm{E}$ we detail calculations involving the primary fields. In appendix $\mathrm{F}$ we translate the current-current OPEs into (anti-)commutation relations for the modes of the currents when the theory is defined on a cylinder. Finally, classical integrability of the model is proven in appendix $\mathrm{G}$.

\section{The conformal current algebra}

Setting. We study a non-linear sigma-model on a supergroup $G$ with zero Killing form, including a kinetic term and a Wess-Zumino term with arbitrary coefficient. The model is conformal and has a global symmetry group corresponding to the left and right action of the group on itself. In this section we review and complement the analysis of the algebra of current components associated to the left group action [4]. The action of the non-linear sigma-model on the supergroup is:

$$
\begin{aligned}
S & =S_{k i n}+S_{W Z} \\
S_{k i n} & =\frac{1}{16 \pi f^{2}} \int d^{2} z \operatorname{Tr}^{\prime}\left[-\partial^{\mu} g^{-1} \partial_{\mu} g\right] \\
S_{W Z} & =-\frac{i k}{24 \pi} \int_{B} d^{3} y \epsilon^{\alpha \beta \gamma} \operatorname{Tr}^{\prime}\left(g^{-1} \partial_{\alpha} g g^{-1} \partial_{\beta} g g^{-1} \partial_{\gamma} g\right)
\end{aligned}
$$

where $g$ takes values in the supergroup $G$ and $T r^{\prime}$ indicates the non-degenerate bi-invariant metric. We will use the normalization and results of [4]. The Wess-Zumino-Witten points are given by the equation $1 / f^{2}=|k|$. Note that the action is invariant under group inversion $g \leftrightarrow g^{-1}$ and simultaneous orientation reversal $z \leftrightarrow \bar{z}$.

The conformal current algebra. From the action (2.1) we can calculate the classical currents associated to the invariance of the theory under left multiplication of the field $g$ by a group element in $G_{L}$ and right multiplication by a group element in $G_{R}$. The classical $G_{L}$ currents are given by

$$
\begin{aligned}
& j_{L, z}=c_{+} \partial g g^{-1} \\
& j_{L, \bar{z}}=c_{-} \bar{\partial} g g^{-1},
\end{aligned}
$$

where the constant $c_{+}$and $c_{-}$are given in terms of the couplings by:

$$
c_{ \pm}=-\frac{\left(1 \pm k f^{2}\right)}{2 f^{2}}
$$

Similarly, we also have the left-invariant currents that generate right multiplication:

$$
\begin{aligned}
& j_{R, z}=-c_{-} g^{-1} \partial g \\
& j_{R, \bar{z}}=-c_{+} g^{-1} \bar{\partial} g .
\end{aligned}
$$


The operator product expansions (OPEs) satisfied by the left currents have been derived in [4]. They read:

$$
\begin{aligned}
& j_{L, z}^{a}(z) j_{L, z}^{b}(0) \sim \kappa^{a b} \frac{c_{1}}{z^{2}}+f^{a b}{ }_{c}\left[\frac{c_{2}}{z} j_{L, z}^{c}(0)+\left(c_{2}-g\right) \frac{\bar{z}}{z^{2}} j_{L, \bar{z}}^{c}(0)\right] \\
&+f^{a b}{ }_{c}\left[-\frac{g}{4} \frac{\bar{z}}{z}\left(\partial_{z} j_{\bar{z}}^{c}(0)-\partial_{\bar{z}} j_{z}^{c}(0)\right)+\frac{c_{2}}{2} \partial_{z} j_{L, z}^{c}(0)+\frac{c_{2}-g}{2} \frac{\bar{z}^{2}}{z^{2}} \partial_{\bar{z}} j_{L, \bar{z}}^{c}(0)\right] \\
&+: j_{z}^{a} j_{z}^{b}:(0)+A^{a b}{ }_{c d} \frac{1}{2} \frac{\bar{z}^{2}}{z^{2}}: j_{\bar{z}}^{c} j_{\bar{z}}^{d}:(0)+B^{a b}{ }_{c d} \frac{\bar{z}}{z}: j_{z}^{c} j_{\bar{z}}^{d}:(0)-C^{a b}{ }_{c d} \log |z|^{2}: j_{z}^{c} j_{z}^{e}:(0) \\
&+\ldots \\
& j_{L, \bar{z}}^{a}(z) j_{L, \bar{z}}^{b}(0) \sim \kappa^{a b} c_{3} \frac{1}{\bar{z}^{2}}+f^{a b}{ }_{c}\left[\frac{c_{4}}{\bar{z}} j_{L, \bar{z}}^{c}(0)+\frac{\left(c_{4}-g\right) z}{\bar{z}^{2}} j_{L, z}^{c}(0)\right] \\
& \quad+f^{a b}{ }_{c}\left[\frac{g}{4} \frac{z}{\bar{z}}\left(\partial_{z} j_{\bar{z}}^{c}(0)-\partial_{\bar{z}} j_{z}^{c}(0)\right)+\frac{c_{4}}{2} \partial_{\bar{z}} j_{L, \bar{z}}^{c}(0)+\frac{c_{4}-g}{2} \frac{z^{2}}{\bar{z}^{2}} \partial_{z} j_{L, z}^{c}(0)\right] \\
&+: j_{\bar{z}}^{a} j_{\bar{z}}^{b}:(0)-A^{a b}{ }_{c d} \log |z|^{2}: j_{\bar{z}}^{c} j_{\bar{z}}^{d}:(0)+B^{a b}{ }_{c d} \frac{z}{\bar{z}}: j_{z}^{c} j_{\bar{z}}^{d}:(0)+C^{a b}{ }_{c d} \frac{1}{2} \frac{z^{2}}{\bar{z}^{2}}: j_{z}^{c} j_{z}^{d}:(0) \\
& \quad+\ldots \\
& j_{L, z}^{a}(z) j_{L, \bar{z}}^{b}(0) \sim \tilde{c} \kappa^{a b} 2 \pi \delta^{(2)}(z-w)+f^{a b}{ }_{c}\left[\frac{\left(c_{4}-g\right)}{\bar{z}} j_{L, z}^{c}(0)+\frac{\left(c_{2}-g\right)}{z} j_{L, \bar{z}}^{c}(0)\right] \\
& \quad+f^{a b}{ }_{c}\left[-\frac{g}{4} \log |z|^{2}\left(\partial_{z} j_{\bar{z}}^{c}(0)-\partial_{\bar{z}} j_{z}^{c}(0)\right)+\frac{\left(c_{4}-g\right) z}{\bar{z}} j_{L, z}^{c}(0)\right] \\
&+: j_{z}^{a} j_{\bar{z}}^{b}:(0)+A^{a b}{ }_{c d} \frac{\bar{z}}{z}: j_{\bar{z}}^{c} j_{\bar{z}}^{d}:(0)-B^{a b}{ }_{c d} \log |z|^{2}: j_{z}^{c} j_{\bar{z}}^{d}:(0)+C^{a b}{ }_{c d} \frac{z}{\bar{z}}: j_{z}^{c} j_{z}^{d}:(0) \\
& \quad+\ldots
\end{aligned}
$$

Compared to [4], we have added a few terms at order zero in the distance between the insertion points of the two current components. ${ }^{1}$ The ellipses refer to subleading terms in the expansion in the distance between the two insertion points (which includes logarithms). The right current components $j_{R, z}$ and $j_{R, \bar{z}}$ satisfy similar operator product expansions amongst themselves, with the holomorphic coordinates replaced by anti-holomorphic ones. This can be proven by using the $\mathbb{Z}_{2}$ symmetry that we noted before. Associativity of the current algebra is discussed in appendix D.4.

For the supergroup non-linear sigma-model in equation (2.1), the coefficients of the second and first order poles in the conformal current algebra, expressed purely in terms of $c_{ \pm}$, are given by [4]

$$
\begin{aligned}
c_{1} & =-\frac{c_{+}^{2}}{c_{+}+c_{-}} & c_{3} & =-\frac{c_{-}^{2}}{c_{+}+c_{-}} \\
c_{2} & =i \frac{c_{+}\left(c_{+}+2 c_{-}\right)}{\left(c_{+}+c_{-}\right)^{2}} & c_{4} & =i \frac{c_{-}\left(2 c_{+}+c_{-}\right)}{\left(c_{+}+c_{-}\right)^{2}} \\
g & =i \frac{2 c_{+} c_{-}}{\left(c_{+}+c_{-}\right)^{2}} & \tilde{c} & =\frac{c_{+} c_{-}}{c_{+}+c_{-}},
\end{aligned}
$$

\footnotetext{
${ }^{1}$ We would like to thank Anatoly Konechny for stressing the importance of these terms, and for sharing his insights in these terms in perturbation theory near Wess-Zumino-Witten points.
} 
where the coefficients $c_{ \pm}$are the factors defined in equation (2.3) as the normalization of the currents. The coefficients $c_{i}$ are exact.

The current algebra defined by equation (2.5) is compatible with both current conservation and the Maurer-Cartan equation:

$$
\begin{aligned}
\bar{\partial} j_{L, z}^{a}+\partial j_{L, \bar{z}}^{a} & =0 \\
c_{-} \bar{\partial} j_{L, z}^{a}-c_{+} \partial j_{L, \bar{z}}^{a}-i f_{b c}^{a}: j_{L, z}^{c} j_{L, \bar{z}}^{b}: & =0 .
\end{aligned}
$$

Indeed the OPE of a current with the left-hand side of the current conservation equation (2.7) (respectively the Maurer-Cartan equation (2.8)) gives zero up to contact terms (respectively exactly zero). Moreover, demanding compatibility of the current algebra with both equations (2.7) and (2.8) is a way to determine all the other subleading terms in the current algebra, order by order in semi-classical perturbation theory, namely for small $f^{2}$ (at fixed $k f^{2}$ ). This is explained in section 5. As we will see, the assumption of the validity of current conservation and especially the Maurer-Cartan equation in the quantum theory, determines a tightly constrained and interesting algebraic structure associated to supergroups with vanishing Killing form. This hypothesis is tightly linked to the quantum integrability of the model, as we discuss in section 7 .

We can use this perturbative technique to compute the coefficients of the current bilinears that appear in equation (2.5), up to order $f^{2}$. This computation is detailed in appendix D.1 and it leads to the results:

$$
\begin{aligned}
A^{a b}{ }_{c d} & =\frac{c_{+}^{2}}{\left(c_{+}+c_{-}\right)^{3}} \frac{1}{2}\left(f_{c g}^{b} f_{d}^{a g}(-1)^{c d}+f_{d g}^{b} f_{c}^{a g}\right)+\mathcal{O}\left(f^{4}\right) \\
B^{a b}{ }_{c d} & =\frac{c_{+} c_{-}}{\left(c_{+}+c_{-}\right)^{3}}\left(f^{b}{ }_{c g} f_{d}^{a g}(-1)^{c d}+f_{d g}^{b} f_{c}^{a g}{ }_{c}\right)+\mathcal{O}\left(f^{4}\right) \\
C^{a b}{ }_{c d} & =\frac{c_{-}^{2}}{\left(c_{+}+c_{-}\right)^{3}} \frac{1}{2}\left(f^{b}{ }_{c g} f_{d}^{a g}(-1)^{c d}+f_{d g}^{b} f^{a g}{ }_{c}\right)+\mathcal{O}\left(f^{4}\right) .
\end{aligned}
$$

The fact that the same tensors appear in the three different current-current OPEs (2.5) is a consequence of current conservation. The four-tensors $A, B, C$ are (graded) symmetric in their two upper indices. This follows from the interchangeability of the current components on the left hand side of the first two OPEs in (2.5). Equation (2.9) shows that these fourtensors are also (graded) symmetric in their two lower indices. Thus they are linear maps from graded symmetric tensors onto graded symmetric tensors. They partially code higher order corrections to equation (2.9) (see appendix D.1).

In appendix D.1, we have included a careful discussion of minus signs arising due to the graded statistics of the supergroup. For the remainder of the paper however, we will not be careful about minus signs arising due to the grading of operators. Since we use only universal group and (super) Lie algebra properties in our calculations, all signs can be consistently restored.

The Virasoro algebra from the current algebra. In [4] it was shown that the left and right Virasoro algebra emerge from the current algebra (2.5) via the Sugawara construction. For instance the holomorphic stress-tensor:

$$
T(z)=\frac{1}{2 c_{1}} \kappa_{b a}: j_{L, z}^{a} j_{L, z}^{b}:
$$


satisfies the following OPEs:

$$
\begin{aligned}
T(z) j_{L, z}^{a}(w) & =\frac{j_{L, z}^{a}(w)}{(z-w)^{2}}+\frac{\partial j_{L, z}^{a}(w)}{z-w}+\mathcal{O}(z-w)^{0} \\
T(z) j_{L, \bar{z}}^{a}(w) & =\frac{\partial j_{L, \bar{z}}^{a}(w)}{z-w}+\mathcal{O}(z-w)^{0} \\
T(z) T(w) & =\frac{\operatorname{sdim}(G)}{2(z-w)^{4}}+\frac{T(w)}{(z-w)^{2}}+\frac{\partial T(w)}{z-w}+\mathcal{O}(z-w)^{0} .
\end{aligned}
$$

In appendix D.2 we give more details of the proof of equation (2.11). In particular it is shown that the terms of order zero in equation (2.5) (as well as any of the other subleading terms) do not modify this OPE. We also checked through explicit computation that the invariant contractions of the structure constants and the metric with the four-tensors (2.9) appearing in the energy-momentum tensor/current OPE give zero for the algebra $p s l(2 \mid 2)$.

\section{The left-right current algebra}

In this section, we compute the operator product expansions of currents associated to the left and the right action of the group upon itself.

\subsection{The primary adjoint operator}

The right current components can be rewritten in terms of the adjoint group action on the left currents:

$$
\begin{aligned}
& j_{R, z}=-c_{-} g^{-1} \partial g=-\frac{c_{-}}{c_{+}} A d_{g^{-1}}\left(j_{L, z}\right) \\
& j_{R, \bar{z}}=-c_{+} g^{-1} \bar{\partial} g=-\frac{c_{+}}{c_{-}} A d_{g}\left(j_{L, \bar{z}}\right) .
\end{aligned}
$$

In the quantum theory the adjoint group action is generated by an operator that we call the primary adjoint operator:

$$
\mathcal{A}^{a \bar{a}}=x \operatorname{Str}\left(g^{-1} t^{a} g t^{\bar{a}}\right) .
$$

Here $x$ is some normalization factor. This operator transforms in the adjoint representation with respect to both the left and the right algebras. In the following unbarred (respectively barred) indices refer to the left (respectively right) adjoint representation. We recall that this field is also useful in writing down the Lagrangian of the model, and that its anomalous dimension is proportional to the beta-function of the model (which is zero in the case under study) [16]. Special properties of the primary adjoint operator in non-linear sigma models on supergroup with vanishing Killing form were also discussed in [9]. We can rewrite equations (3.1) and (3.2) as:

$$
\begin{gathered}
j_{R, z}^{\bar{b}}=-\frac{c_{-}}{c_{+}} \kappa_{b a}: j_{L, z}^{a} \mathcal{A}^{b \bar{b}}: \\
j_{R, \bar{z}}^{\bar{b}}=-\frac{c_{+}}{c_{-}} \kappa_{b a}: j_{L, \bar{z}}^{a} \mathcal{A}^{b \bar{b}}: .
\end{gathered}
$$


Using the $\mathbb{Z}_{2}$ symmetry of the theory we have also:

$$
\begin{aligned}
& j_{L, z}^{b}=-\frac{c_{+}}{c_{-}} \kappa_{\bar{b} \bar{a}}: j_{R, z}^{\bar{a}} \mathcal{A}^{b \bar{b}}:(z) \\
& j_{L, \bar{z}}^{b}=-\frac{c_{-}}{c_{+}} \kappa_{\bar{b} \bar{a}}: j_{R, \bar{z}}^{\bar{a}} \mathcal{A}^{b \bar{b}}:(z)
\end{aligned}
$$

These relations fix a normalization for the operator $\mathcal{A}^{a \bar{a}}$. They are compatible if the following relations hold:

$$
\begin{aligned}
\kappa_{b a} \mathcal{A}^{a \bar{a}} \mathcal{A}^{b \bar{b}} & =\kappa^{\bar{a} \bar{b}} I \\
\kappa_{\bar{b} \bar{a}} \mathcal{A}^{a \bar{a}} \mathcal{A}^{b \bar{b}} & =\kappa^{a b} I
\end{aligned}
$$

where $I$ is the identity at least as acting upon the current algebra. One can argue more generically that these bilinears are proportional to the unit operator by using the definition of the primary adjoint in terms of the supertrace, and using completeness of the Lie algebra generators. Remember also that the left and right conformal dimensions of the adjoint operator $\mathcal{A}^{a \bar{a}}$ vanish since they are proportional to the dual Coxeter number of the Lie superalgebra.

The action of the zero modes of the currents generates the group transformations. Since the structure constants are the generators of the Lie superalgebra in the adjoint representation, the OPE between a current and the primary adjoint operator reads:

$$
\begin{aligned}
j_{L, z}^{a}(z) \mathcal{A}^{b \bar{b}}(w) & =\frac{c_{+}}{c_{+}+c_{-}} \frac{i f_{{ }_{c}}^{a b} \mathcal{A}^{c \bar{b}}}{z-w}+\ldots \\
j_{L, \bar{z}}^{a}(z) \mathcal{A}^{b \bar{b}}(w) & =\frac{c_{-}}{c_{+}+c_{-}} \frac{i f^{a b}{ }_{{ }^{\prime}} \mathcal{A}^{c \bar{b}}}{\bar{z}-\bar{w}}+\ldots \\
j_{R, z}^{\bar{a}}(z) \mathcal{A}^{b \bar{b}}(w) & =\frac{c_{-}}{c_{+}+c_{-}} \frac{i f^{\bar{a} \bar{b}}{ }_{\bar{c}} \mathcal{A}^{b \bar{c}}}{z-w}+\ldots \\
j_{R, \bar{z}}^{\bar{a}}(z) \mathcal{A}^{b \bar{b}}(w) & =\frac{c_{+}}{c_{+}+c_{-}} \frac{i f^{\bar{a} \bar{b}} \mathcal{A}_{\bar{c}}^{b \bar{c}}}{\bar{z}-\bar{w}}+\ldots
\end{aligned}
$$

In section 4 the concept of primary field will be defined precisely. The coefficients appearing in the previous OPE will be explained, and we will compute the first subleading terms (see equation (4.2)).

Moreover, we propose that the following equations hold in the model under consideration:

$$
\begin{aligned}
\partial \mathcal{A}^{a \bar{a}} & =-\frac{i f^{a}{ }_{b c}}{c_{+}}: j_{L, z}^{c} \mathcal{A}^{b \bar{a}}:=-\frac{i f^{\bar{a}_{\bar{b} \bar{c}}}}{c_{-}}: j_{R, z}^{\bar{c}} \mathcal{A}^{a \bar{b}}: \\
\bar{\partial} \mathcal{A}^{a \bar{a}} & =-\frac{i f^{a}{ }_{b c}}{c_{-}}: j_{L, \bar{z}}^{c} \mathcal{A}^{b \bar{a}}:=-\frac{i f^{\bar{a}}{ }_{\bar{b} \bar{c}}}{c_{+}}: j_{R, \bar{z}}^{\bar{c}} \mathcal{A}^{a \bar{b}}:
\end{aligned}
$$

One argument for the previous equations is the following. We start with the definition of the adjoint operator in terms of the group element (3.3), and compute its derivative:

$$
\begin{aligned}
\partial \mathcal{A}^{a \bar{a}} & =x \partial \operatorname{STr}\left(g^{-1} t^{a} g t^{\bar{a}}\right) \\
& =x \operatorname{STr}\left(-g^{-1} \partial g g^{-1} t^{a} g t^{\bar{a}}+g^{-1} t^{a} \partial g g^{-1} g t^{\bar{a}}\right)
\end{aligned}
$$




$$
\begin{aligned}
& =x \frac{j_{L, z}^{c} \kappa_{d c}}{c_{+}} \operatorname{STr}\left(g^{-1}\left[t^{a}, t^{d}\right] g t^{\bar{a}}\right) \\
& =-\frac{j_{L, z}^{c}}{c_{+}} i f_{b c}^{a} \mathcal{A}^{b \bar{a}} .
\end{aligned}
$$

We have left out the normal ordering symbols from the above classical calculation. The properties used in the calculation are that the supertrace is graded cyclic and the fact that the equation $g g^{-1}=1$ and its derivative hold true. We assume that the quantum theory is consistent with these two rules. In section 4 we will give a generic proof of equations (3.11) and (3.12), valid up to a certain order in a semi-classical expansion (see equation (4.10)).

Notice that the relations (3.11) and (3.12) imply that $\partial\left(\kappa_{a b} \mathcal{A}^{a \bar{a}} \mathcal{A}^{b \bar{b}}\right)=0=$ $\bar{\partial}\left(\kappa_{a b} \mathcal{A}^{a \bar{a}} \mathcal{A}^{b \bar{b}}\right)$ (and identical equations with the barred indices contracted), and thus are compatible with the equations relating the adjoint primary to the identity (3.8) and (3.9).

\subsection{The left current - right current OPEs}

We have collected the tools to calculate the left/right current operator product expansions. Thanks to equations (3.4) and (3.5) we only need the left current self OPEs (2.5) as well as the OPE between the left current and the adjoint primary operator (3.10). As an example, we will explicitly compute the OPE $j_{L, z}^{a}(z) j_{R, z}^{\bar{a}}(w)$ at the order of the poles. We use the prescription of appendix A:

$$
\begin{aligned}
j_{L, z}^{a}(z) j_{R, z}^{\bar{a}}(w)=-j_{L, z}^{a}(z) \frac{c_{-}}{c_{+}} \kappa_{c b}: j_{L, z}^{b} \mathcal{A}^{c \bar{a}}:(w) \\
=-\frac{c_{-}}{c_{+}} \kappa_{c b} \lim _{x \rightarrow w:}\left[j_{L, z}^{a}(z) j_{L, z}^{b}(x) \mathcal{A}^{c \bar{a}}(w)\right] \\
=-\frac{c_{-}}{c_{+}} \kappa_{c b} \lim _{x \rightarrow w:}\left[\left(\frac{c_{1} \kappa^{a b}}{(z-x)^{2}}+\frac{c_{2} f^{a b}{ }_{d j_{L, z}}^{d}(x)}{z-x}+\frac{\left(c_{2}-g\right) f^{a b}{ }_{d} j_{L, \bar{z}}^{d}(x)(\bar{z}-\bar{x})}{(z-x)^{2}}+\ldots\right) \mathcal{A}^{c \bar{a}}(w)\right. \\
\left.\quad+j_{L, z}^{b}(x)\left(\frac{c_{+}}{c_{+}+c_{-}} \frac{i f^{a c}{ }_{d} \mathcal{A}^{d \bar{a}}(w)}{z-w}+\ldots\right)\right] \\
=-\frac{c_{-}}{c_{+}}\left[\frac{c_{1} \mathcal{A}^{a \bar{a}}(w)}{(z-w)^{2}}+\left(-c_{2}+\frac{i c_{+}}{c_{+}+c_{-}}\right) \frac{f_{b c}^{a}: j_{L, z}^{b} \mathcal{A}^{c \bar{a}}:(w)}{z-w}\right. \\
\left.\quad-\left(c_{2}-g\right) \frac{f_{b c}^{a}: j_{L, \bar{z}}^{b} \mathcal{A}^{c \bar{a}}:(w)(\bar{z}-\bar{w})}{(z-w)^{2}}+\ldots\right]
\end{aligned}
$$

In principle the second- and first-order poles that we obtain in the last line may receive corrections from the lower-order terms that we neglected in the penultimate line. We will now argue that it is not the case. Let us consider the first term in the last line (the second-order pole). This term may receive corrections of the form $T_{b}^{a} \mathcal{A}^{b \bar{a}}$, where the tensor $T^{a}{ }_{b}$ contains at least one structure constant. Such a tensor vanishes by using properties of the Lie super algebras under consideration [2]. Let us now consider the second term (the holomorphic simple pole). It could receive corrections of the form $T_{b c}^{a}: j_{L, z}^{b} \mathcal{A}^{c \bar{a}}$, where $T_{b c}^{a}$ contains at least two structure constants. Again, according to [2], this tensor vanishes because traceless four-tensors invariantly contracted with structure constants over two indices vanish. The third term receives no higher order corrections for the same reason. 
Thus the terms written in the last line of (3.14) are not corrected. Using equations (3.11) and (3.12) we finally obtain:

$j_{L, z}^{a}(z) j_{R, z}^{\bar{a}}(w)=\frac{c_{+} c_{-}}{c_{+}+c_{-}}\left(\frac{\mathcal{A}^{a \bar{a}}(w)}{(z-w)^{2}}+\frac{c_{-}}{c_{+}+c_{-}} \frac{\partial \mathcal{A}^{a \bar{a}}(w)}{z-w}+\frac{c_{-}}{c_{+}+c_{-}} \frac{\bar{\partial} \mathcal{A}^{a \bar{a}}(w)(\bar{z}-\bar{w})}{(z-w)^{2}}\right)+\ldots$

where the ellipses refer to terms of order zero or more in the distance between the two operators. Similarly we can compute:

$$
\begin{aligned}
& j_{L, \bar{z}}^{a}(z) j_{R, \bar{z}}^{\bar{a}}(w)=\frac{c_{+} c_{-}}{c_{+}+c_{-}}\left(\frac{\mathcal{A}^{a \bar{a}}(w)}{(\bar{z}-\bar{w})^{2}}+\frac{c_{+}}{c_{+}+c_{-}} \frac{\partial \mathcal{A}^{a \bar{a}}(w)(z-w)}{(\bar{z}-\bar{w})^{2}}+\frac{c_{+}}{c_{+}+c_{-}} \frac{\bar{\partial} \mathcal{A}^{a \bar{a}}(w)}{\bar{z}-\bar{w}}\right)+\ldots \\
& j_{L, z}^{a}(z) j_{R, \bar{z}}^{\bar{a}}(w)=-\frac{c_{+}^{2}}{c_{+}+c_{-}}\left(\mathcal{A}^{a \bar{a}}(w) 2 \pi \delta^{(2)}(z-w)-\frac{c_{-}}{c_{+}+c_{-}} \frac{\partial \mathcal{A}^{a \bar{a}}(w)}{\bar{z}-\bar{w}}+\frac{c_{-}}{c_{+}+c_{-}} \frac{\bar{\partial} \mathcal{A}^{a \bar{a}}(w)}{z-w}\right)+\ldots \\
& j_{L, \bar{z}}^{a}(z) j_{R, z}^{\bar{a}}(w)=-\frac{c_{-}^{2}}{c_{+}+c_{-}}\left(\mathcal{A}^{a \bar{a}}(w) 2 \pi \delta^{(2)}(z-w)+\frac{c_{+}}{c_{+}+c_{-}} \frac{\partial \mathcal{A}^{a \bar{a}}(w)}{\bar{z}-\bar{w}}-\frac{c_{+}}{c_{+}+c_{-}} \frac{\bar{\partial} \mathcal{A}^{a \bar{a}}(w)}{z-w}\right)+\ldots
\end{aligned}
$$

The first two OPEs can be written in the alternative form:

$$
\begin{aligned}
& j_{L, z}^{a}(z) j_{R, z}^{\bar{a}}(w)=\frac{c_{+} c_{-}}{c_{+}+c_{-}} \frac{\mathcal{A}^{a \bar{a}}(w)\left(\frac{c_{+} w+c_{-} z}{c_{+}+c_{-}}\right)}{(z-w)^{2}}+\ldots \\
& j_{L, \bar{z}}^{a}(z) j_{R, \bar{z}}^{\bar{a}}(w)=\frac{c_{+} c_{-}}{c_{+}+c_{-}} \frac{\mathcal{A}^{a \bar{a}}(w)\left(\frac{c_{-} w+c_{+} z}{c_{+}+c_{-}}\right)}{(\bar{z}-\bar{w})^{2}}+\ldots
\end{aligned}
$$

It is straightforward to show that the OPEs are compatible with current conservation and the Maurer-Cartan equation. These OPEs are also compatible with the fact that the stresstensor can be written either in terms of the left-current or in terms of the right currents. As an example of these consistency checks, it is shown in appendix D.3 that when we express the energy-momentum tensor in terms of right currents, it satisfies the expected OPE with the left current:

$$
T(z) j_{L, z}^{a}(w)=\frac{1}{2 c_{3}} \kappa_{\bar{c} \bar{b}}: j_{R, z}^{\bar{b}} j_{R, z}^{\bar{c}}:(z) j_{L, z}^{a}(w)=\frac{j_{L, z}^{a}(w)}{(z-w)^{2}}+\frac{\partial j_{L, z}^{a}(w)}{z-w}+\mathcal{O}\left((z-w)^{0}\right)
$$

When the theory is defined on a cylinder we can Fourier expand the currents along the angular coordinate, at a given time. It was shown in [4] that the modes of the time components of the left (or the right) currents generate an affine Lie algebra at level $k$. The full commutator algebra computed in appendix $\mathrm{F}$ shows that these two affine Lie algebras commute.

Summary. In this section we have determined the pole order parts of the left and right current operator product expansions. The algebra closes on the current components and the adjoint field. Under the assumptions on the quantum theory stated above, the coefficients of the algebra are exact. ${ }^{2}$ We now move from the determination of the left-right symmetry algebra of the model to the study of the vertex operators.

\footnotetext{
${ }^{2}$ If the assumptions are not valid, the coefficients will receive higher order corrections in $f^{2}$. The results in the rest of the paper are independent of these possible corrections.
} 


\section{The primaries}

In this section we define the concept of current algebra primaries. These fields can be understood as the elementary vertex operators of the conformal field theory. We compute the operator product expansion between a primary field and a current perturbatively, and deduce the OPE between a primary field and the stress-tensor. In particular we derive the OPEs used in [15].

Left current algebra primaries. Given a representation $\mathcal{R}$ of the group $G_{L}$ we define a left primary field $\phi_{\mathcal{R}}$ with respect to the left current algebra (2.5) as a field satisfying the operator product expansions:

$$
\begin{aligned}
& j_{L, z}^{a}(z, \bar{z}) \phi_{\mathcal{R}}(w, \bar{w})=-\frac{c_{+}}{c_{+}+c_{-}} t^{a} \frac{\phi_{\mathcal{R}}(w, \bar{w})}{z-w}+\text { order zero } \\
& j_{L, \bar{z}}^{a}(z, \bar{z}) \phi_{\mathcal{R}}(w, \bar{w})=-\frac{c_{-}}{c_{+}+c_{-}} t^{a} \frac{\phi_{\mathcal{R}}(w, \bar{w})}{\bar{z}-\bar{w}}+\text { order zero }
\end{aligned}
$$

where the matrices $t^{a}$ are the generators of the Lie super-algebra taken in the representation $\mathcal{R}$ associated to the primary field $\phi_{\mathcal{R}}$. If one assumes the above form for the operator product expansions, then the coefficients of the poles are fixed by the Ward identity for the symmetry $G_{L}$ and the demand that the contact term vanishes in the operator product expansion between the field $\phi$ and the Maurer-Cartan operator (2.8). The Ward identity implies compatibility of the OPEs (4.1) with current conservation (2.7). An example of a left current primary is the adjoint primary we discussed in the previous section.

In appendix E.1 it is shown that a current primary field at a given point of the moduli space remains a current primary field after deformation of the kinetic term in the action. Thus one can consistently think of the current algebra primaries as the group element $g$ taken in the representation $\mathcal{R}$. It also implies that at the WZW points the current primaries are the affine primary fields.

As argued in section 5, we can compute the less singular terms in the current-primary OPE (4.1) order by order in $f^{2}$, by using the current conservation and the Maurer-Cartan equation. Performing the calculation of higher order terms to order $f^{2}$, we find the OPE:

$$
\begin{aligned}
j_{L, z}^{a}(z, \bar{z}) \phi(w, \bar{w})= & -\frac{c_{+}}{c_{+}+c_{-}} t^{a} \frac{\phi(w, \bar{w})}{z-w}+: j_{L, z}^{a} \phi:(w, \bar{w}) \\
& +A^{a}{ }_{c} \log |z-w|^{2}: j_{L, z}^{c} \phi:(w, \bar{w})+B^{a}{ }_{c} \frac{\bar{z}-\bar{w}}{z-w}: j_{L, \bar{z}}^{c} \phi:(w, \bar{w})+\ldots \\
j_{L, \bar{z}}^{a}(z, \bar{z}) \phi(w, \bar{w})= & -\frac{c_{-}}{c_{+}+c_{-}} t^{a} \frac{\phi(w, \bar{w})}{\bar{z}-\bar{w}}+: j_{L, \bar{z}}^{a} \phi:(w, \bar{w}) \\
& -A^{a}{ }_{c} \frac{z-w}{\bar{z}-\bar{w}}: j_{L, z}^{c} \phi:-B^{a}{ }_{c} \log |z-w|^{2}: j_{L, \bar{z}}^{c} \phi:(w, \bar{w})+\ldots
\end{aligned}
$$

where we dropped the subscript $\mathcal{R}$. The coefficients read:

$$
A_{c}^{a}=\frac{c_{-}}{\left(c_{+}+c_{-}\right)^{2}} i f^{a}{ }_{c b} t^{b}+\mathcal{O}\left(f^{4}\right) ; \quad B_{c}^{a}=\frac{c_{+}}{\left(c_{+}+c_{-}\right)^{2}} i f_{c b}^{a} t^{b}+\mathcal{O}\left(f^{4}\right) .
$$

The details of the calculation are given in appendix E.2. Note that the coefficients of the simple poles are unmodified. 
Current primaries are Virasoro primaries. We will now show that a primary field with respect to the left-current algebra is also a primary field with respect to the Virasoro algebra. The holomorphic worldsheet stress tensor is:

$$
T(z)=\frac{1}{2 c_{1}} \kappa_{b a}: j_{L, z}^{a} j_{L, z}^{b}:(z) .
$$

Let us consider the OPE between a left-primary field $\phi$ and the holomorphic stress-tensor:

$$
\phi(z) 2 c_{1} T(w)=\lim _{: x \rightarrow w:} \phi(z) j_{L, z}^{a}(x) j_{L, z}^{b}(w) \kappa_{b a} .
$$

From the structure of the OPE (4.2), and from the fact that all operators appearing in this OPE are assumed to be composites of currents and of the operator $\phi$, it follows that the most singular term that may appear in this OPE is a double pole, multiplying the operator $\phi$. As a consequence all the positive modes $L_{n>0}$ of the holomorphic stress-tensor annihilate the operator $\phi$. Thus this operator is a Virasoro primary.

Furthermore, with the knowledge of the current-primary OPE (4.2) up to order $f^{2}$, we can evaluate the stress-tensor/primary OPE up to the same order. Details about this computation are given in appendix E.3. We obtain:

$$
T(w) \phi(z)=\frac{f^{2}}{2} \frac{t^{a} t^{b} \kappa_{b a} \phi(z)}{(z-w)^{2}}+\frac{1}{c_{+}} \frac{\kappa_{b a} t^{a}: j_{L, z}^{b} \phi:(z)}{w-z}+\mathcal{O}(z-w)^{0}+\mathcal{O}\left(f^{4}\right) .
$$

The same computation can be performed with the anti-holomorphic stress-tensor. We obtain:

$$
\bar{T}(\bar{w}) \phi(z)=\frac{f^{2}}{2} \frac{t^{a} t^{b} \kappa_{b a} \phi(z)}{(\bar{z}-\bar{w})^{2}}+\frac{1}{c_{-}} \frac{\kappa_{b a} t^{a}: j_{L, \bar{z}}^{b} \phi:(z)}{\bar{w}-\bar{z}}+\mathcal{O}(\bar{z}-\bar{w})^{0}+\mathcal{O}\left(f^{4}\right) .
$$

On general grounds the OPE between the stress-tensor and the primary field $\phi$ reads:

$$
T(w) \phi(z)=\frac{\Delta_{\phi} \phi(z)}{(w-z)^{2}}+\frac{\partial \phi(z)}{w-z}+\mathcal{O}\left((z-w)^{0}\right),
$$

where $\Delta_{\phi}$ is the left conformal dimension of the operator $\phi$. Thus we deduce the conformal dimensions of the primary field $\phi$ :

$$
\Delta_{\phi}=\bar{\Delta}_{\phi}=\frac{f^{2}}{2} t^{a} t^{b} \kappa_{b a}+\mathcal{O}\left(f^{4}\right)
$$

The semi-classical result for the conformal dimension of a current primary is as expected. It is equal to the quadratic Casimir of the representation in which the field transforms, times the inverse radius of the group manifold squared. For generic current primaries, there could be corrections of order $f^{4}$ to this formula. These corrections were conjectured to be absent in [2]. This was proven to be the case to all orders in perturbation theory if the superdimension of the representation of the primary is non-zero (i.e. for short multiplets). For example for the short, discrete representation crucial to the calculation in [15], there are no corrections. 
Notice that the stress-energy tensor can also be written in terms of the right currents. Equation (4.9) implies that a primary field transforms under the left- and right-action of the group in representations that have the same eigenvalue of the quadratic Casimir operator. The simple poles in (4.6) and (4.7) also give the relations:

$$
\begin{aligned}
& \partial \phi(z)=\frac{1}{c_{+}} \kappa_{b a} t^{a}: j_{L, z}^{b} \phi:(z)+\mathcal{O}\left(f^{4}\right) \\
& \bar{\partial} \phi(z)=\frac{1}{c_{-}} \kappa_{b a} t^{a}: j_{L, \bar{z}}^{b} \phi:(z)+\mathcal{O}\left(f^{4}\right) .
\end{aligned}
$$

Remark about the atypical sector. Some of the primary fields are associated to atypical Kac modules, that are reducible but indecomposable [7]. In that case the matrices $t^{a}$ that appear in equation (4.1) are not invertible. Moreover the quadratic operator $\kappa_{b a} t^{a} t^{b}$ can then be written in an upper-triangular form, with zeros on the diagonal (which is the generalized eigenvalue of the quadratic casimir for atypical representations of e.g. the $\operatorname{psl}(n \mid n)$ superalgebra). Equation (4.6) tells us that the operator $L_{0}$ is proportional to this quadratic operator $\kappa_{b a} t^{a} t^{b}$ when acting on a primary field. This implies that $L_{0}$ is nondiagonalizable, which betrays the logarithmic nature of the theory (see [7] for a similar argument in the case of $\operatorname{psl}(2 \mid 2)$, and $[18,19]$ for an introduction to logarithmic CFTs). Let us remark here that the fact that the current component $j_{L, z}$ has dimensions $(1,0)$, but is not holomorphic also codes the logarithmic nature of the conformal field theory [8].

\section{A recursive bootstrap for the elementary operator algebra}

In this section we will explain how to compute the current-current and current-primary OPEs order by order in a semi-classical expansion. We will show that the knowledge of the poles in these OPEs is enough to fix all the subleading terms. The idea driving the bootstrap is to ask for the compatibility of the elementary OPEs with both current conservation and the Maurer-Cartan equation.

Current-current OPEs. First let us consider the current-current OPEs. Current conservation gives the first constraints:

$$
\begin{aligned}
& j_{L, z}^{a}(z)\left[\bar{\partial} j_{L, z}^{b}(w)+\partial j_{L, \bar{z}}^{b}(w)\right]=0 \\
& j_{L, \bar{z}}^{a}(z)\left[\bar{\partial} j_{L, z}^{b}(w)+\partial j_{L, \bar{z}}^{b}(w)\right]=0
\end{aligned}
$$

The first line implies a one-to-one correspondence between the terms in the $j_{L, z}^{a} j_{L, z}^{b}$ and $j_{L, z}^{a} j_{L, \bar{z}}^{b}$ OPEs. The second line then links the $j_{L, \bar{z}}^{a} j_{L, \bar{z}}^{b}$ and the $j_{L, z}^{a} j_{L, \bar{z}}^{b}$ OPEs. These OPEs are expected to vanish up to contact terms. Indeed the same OPEs code the Ward identity for the global symmetry $G_{L}$. It follows that the contact terms in these OPEs are given by the transformation properties of the left current under the left action of the group on itself. ${ }^{3}$

\footnotetext{
${ }^{3}$ These contact terms allow for the computation of the holomorphic (respectively anti-holomorphic) poles in the $j_{L, z}^{a} j_{L, z}^{b}\left(\right.$ respectively $\left.j_{L, \bar{z}}^{a} j_{L, \bar{z}}^{b}\right)$ OPE. These poles were already computed to all orders in $f^{2}$ in [4] using different methods.
} 
The second constraint comes from the Maurer-Cartan equation:

$$
j_{L, z}^{a}(z)\left[c_{-} \bar{\partial} j_{L, z}^{b}(w)-c_{+} \partial j_{L, \bar{z}}^{b}(w)+i f_{c d}^{b}: j_{L, z}^{d} j_{L, \bar{z}}^{c}:(w)\right]=0
$$

Contact terms in this OPE should vanish. Using current conservation and the fact that $c_{+}+c_{-}=-f^{-2}$ we rewrite this constraint as:

$$
j_{L, z}^{a}(z) \bar{\partial} j_{L, z}^{b}(w)=f^{2} j_{L, z}^{a}(z) i f_{c d}^{b}: j_{L, z}^{d} j_{L, \bar{z}}^{c}:(w)
$$

Thanks to the factor of $f^{2}$ on the right-hand side of the previous equation, it becomes manifest that the knowledge of the current algebra at a given order in $f^{2}$ will also determine the current algebra at the next order. The discussion of appendix B shows that the terms appearing in the current-current OPEs at order $f^{2 n}$ are composites of at most $n+1$ currents. This allows us to make an ansatz for the current-current OPE at higher-order. Then equation (5.3) fixes the coefficients in this ansatz. This method is illustrated in appendix D.1 where we compute the current algebra up to order $f^{2}$.

Current-primary OPEs. The same logic applies to the computation of the currentprimary OPEs. Current conservation links the $j_{L, z}^{a} \phi$ and $j_{L, \bar{z}}^{a} \phi$ OPEs:

$$
\phi(z)\left[\bar{\partial} j_{L, z}^{a}(w)+\partial j_{L, \bar{z}}^{a}(w)\right]=0
$$

When the above equation is valid, the Maurer-Cartan constraint can be rewritten as:

$$
\phi(z) \bar{\partial} j_{L, z}^{a}(w)=f^{2} \phi(z) i f_{c d}^{a}: j_{L, z}^{d} j_{L, \bar{z}}^{c}:(w)
$$

Again the discussion of appendix B gives an ansatz for the current-primary OPE at a given order in $f^{2}$ : the terms appearing in the current-primary OPE at order $f^{2 n}$ are composites of at most $n$ currents with the primary field $\phi$. When we plug this ansatz in equation (5.5) we obtain the value of the coefficients. This method is illustrated in appendix E.2 where we compute the current-primary OPE up to order $f^{2}$.

Further remarks. This perturbative approach squares well with the observation that the most singular terms in the current-current and current-primary OPEs come with the lower power of $f^{2}$. This is explained in appendix B. Thus performing a computation up to a certain order in $f^{2}$ allows to truncate the current-current and current-primary OPEs at a certain order in the distance between the insertion points of the operators.

The consistency of this perturbative approach demands that the addition of higherorder terms to the elementary OPEs does not spoil their compatibility both with current conservation and with the Maurer-Cartan equation at lower order in $f^{2}$. That this is the case is proven in appendix $\mathrm{C}$.

One may hope to obtain a closed formula for the full current-current and currentprimary OPEs thanks to this algebraic bootstrap. 


\section{Composite operators and their conformal dimension}

In this section we consider operators that are composites of one or more currents with a primary operator. We are mostly interested in the computation of the conformal dimension of such operators as a function of the two parameters $(k, f)$ of the supergroup sigma-model. At the WZW point these operators are descendants in the highest-weight representations of the left affine Lie algebra.

Operators of the form : $\boldsymbol{j}_{L} \boldsymbol{\phi}:$. Let us consider the operator : $j_{L, z}^{a} \phi:$ defined as the regular term in the OPE between the operators $j_{L, z}^{a}$ and $\phi$. To compute the holomorphic dimension of this operator we compute its OPE with the stress-tensor, and look at the second order pole. The computation is done following the method described in appendix A. The fact that the stress-tensor is holomorphic simplifies the calculation. We find:

$$
\begin{aligned}
T(z): j_{L, z}^{a} \phi:(w)= & \lim _{x \rightarrow w:} T(z) j_{L, z}^{a}(x) \phi(w) \\
= & \lim _{x \rightarrow w:}\left\{\left(\frac{j_{L, z}^{a}(x)}{(z-x)^{2}}+\frac{\partial j_{L, z}^{a}(x)}{z-x}\right) \phi(w)+j_{L, z}^{a}(x)\left(\frac{\Delta_{\phi} \phi(w)}{(z-w)^{2}}+\frac{\partial \phi(w)}{z-w}\right)\right\} \\
= & \lim _{: x \rightarrow w:}\left\{\frac { 1 } { ( z - x ) ^ { 2 } } \left(-\frac{c_{+}}{c_{+}+c_{-}} \frac{t^{a} \phi(w)}{x-w}+: j_{L, z}^{a} \phi:(w)\right.\right. \\
& \left.+A^{a}{ }_{c} \log |x-w|^{2}: j_{L, z}^{c} \phi:(w)+B^{a}{ }_{c}{ }^{a} \frac{\bar{x}-\bar{w}}{x-w}: j_{L, \bar{z}}^{c} \phi:(w)+\ldots\right) \\
& +\frac{1}{z-x}\left(\frac{c_{+}}{c_{+}+c_{-}} \frac{t^{a} \phi(w)}{(x-w)^{2}}+: \partial j_{L, z}^{a} \phi:(w)\right. \\
& \left.+A^{a}{ }_{c} \frac{1}{x-w}: j_{L, z}^{c} \phi:(w)-B^{a}{ }_{c} \frac{\bar{x}-\bar{w}}{(x-w)^{2}}: j_{L, \bar{z}}^{c} \phi:(w)+\ldots\right) \\
& \left.+\frac{\Delta_{\phi}: j_{L, z}^{a} \phi:(w)}{(z-w)^{2}}+\frac{: j_{L, z}^{a} \partial \phi:(w)}{z-w}\right\} \\
= & \frac{2}{(z-w)^{3}} \frac{c_{+}}{c_{+}+c_{-}} t^{a} \phi(w)+\frac{: j_{L, z}^{a} \phi:(w)}{(z-w)^{2}} \\
& +\frac{1}{(z-w)^{3}} \frac{c_{+}}{c_{+}+c_{-}} t^{a} \phi(w)+\frac{: \partial j_{L, z}^{a} \phi:(w)}{z-w}+A^{a}{ }_{c} \frac{1}{(z-w)^{2}}: j_{L, z}^{c} \phi:(w) \\
& +\frac{\Delta_{\phi}: j_{L, z}^{a} \phi:(w)}{(z-w)^{2}}+\frac{: j_{L, z}^{a} \partial \phi:(w)}{z-w}+\mathcal{O}(z-w)^{0}
\end{aligned}
$$

Using equation (4.3) we obtain:

$$
\begin{aligned}
T(z): j_{L, z}^{a} \phi:(w)= & -\frac{c_{+}}{c_{+}+c_{-}} \frac{t^{a} \phi(w)}{(z-w)^{3}}+\frac{\left(\Delta_{\phi}+1\right): j_{L, z}^{a} \phi:(w)+\frac{c_{-}}{\left(c_{+}+c_{-}\right)^{2}} i f^{a}{ }_{c b} t^{b}: j_{L, z}^{c} \phi:(w)}{(z-w)^{2}} \\
& +\frac{\partial: j_{L, z}^{a} \phi:(w)}{z-w}+\mathcal{O}\left(f^{4}\right)+\mathcal{O}(z-w)^{0} .
\end{aligned}
$$

The matrices $t^{a}$ are the generators of the Lie algebra in the representation in which the operator $\phi$ transforms. Since one has a non-vanishing third-order pole, not all of the operators : $j_{L, z}^{a} \phi$ : are Virasoro primary. Indeed we know from equation (4.10) that the 
operator $L_{-1} \phi=\partial \phi$, which is a Virasoro descendant, is a linear combination of these operators. However the remaining ones are all Virasoro primaries. In the case where the quadratic Casimir of the representation $\mathcal{R}$ associated to the representation of the operator $\phi$ is non-zero, it is straightforward to check that in the OPE between the stress-tensor and the operator $c_{\mathcal{R}}^{(2)}: j_{L, z}^{a} \phi:-t^{a} t_{b}: j_{L, z}^{b} \phi:$, the third-order pole vanishes. We adopt the notation $c_{\mathcal{R}}^{(2)}$ both for the (generalized) quadratic Casimir operator and for its eigenvalues in the irreducible representation or reducible indecomposable structure $\mathcal{R}$.

From the double pole in equation (6.2) we can read off the action of the scaling operator $L_{0}$ on the operator : $j_{L, z}^{a} \phi::$

$$
L_{0}: j_{L, z}^{a} \phi:=\left(\Delta_{\phi}+1\right): j_{L, z}^{a} \phi:+\frac{c_{-}}{\left(c_{+}+c_{-}\right)^{2}} i f_{c b}^{a} t^{b}: j_{L, z}^{c} \phi: .
$$

The operators : $j_{L, z}^{a} \phi:$ do not diagonalize the scaling operator $L_{0}$. In order to extract the conformal dimensions of these operators we have to compute the eigenvalues of the following operator :

$$
f^{a}{ }_{c b}\left(t^{b}\right)_{\alpha}^{\beta}
$$

where we wrote explicitly the indices $\alpha, \beta$ associated to the representation $\mathcal{R}$ in which the primary field $\phi$ transforms. This operator is an endomorphism acting on the vector space associated to the tensor product of the adjoint and the representation $\mathcal{R}$, namely $\operatorname{Adj} \otimes \mathcal{R}$. Since the structure constants are the generators of the Lie super-algebra in the adjoint representation, the operator (6.4) can be rewritten as:

$$
\kappa_{b d} t_{A d j}^{d} \otimes t_{\mathcal{R}}^{b}
$$

where this time we kept the external indices implicit. The generators of the Lie superalgebra in the (reducible) representation $A d j \otimes \mathcal{R}$ read:

$$
t_{A d j}^{a} \otimes I d+I d \otimes t_{\mathcal{R}}^{a}
$$

Hence the quadratic Casimir operator in the tensor product of representations is:

$$
\begin{aligned}
c_{A d j \otimes \mathcal{R}}^{(2)} & =\kappa_{b a}\left(t_{A d j}^{a} \otimes I d+I d \otimes t_{\mathcal{R}}^{a}\right)\left(t_{A d j}^{b} \otimes I d+I d \otimes t_{\mathcal{R}}^{b}\right) \\
& =c_{\mathcal{R}}^{(2)}+c_{A d j}^{(2)}+2 \kappa_{b a} t_{A d j}^{a} \otimes t_{\mathcal{R}}^{b} .
\end{aligned}
$$

We deduce that the operator (6.4) that we want to diagonalize reads:

$$
\kappa_{b a} t_{A d j}^{a} \otimes t_{\mathcal{R}}^{b}=\frac{1}{2}\left(c_{A d j \otimes \mathcal{R}}^{(2)}-c_{\mathcal{R}}^{(2)}-c_{A d j}^{(2)}\right) .
$$

Recall that the quadratic Casimir vanishes in the adjoint representation: $c_{A d j}^{(2)}=0$. In the tensor product $A d j \otimes \mathcal{R}_{\phi}$, reducible indecomposable structures may appear. The Casimir operator is not diagonalizable on these structures, but we can still define its generalized eigenvalues.

Finally we obtain the conformal dimension of the operators : $j_{L, z}^{a} \phi:$. Let us denote by $\tilde{\mathcal{R}}$ a representation that appears in the tensor product $A d j \otimes \mathcal{R}$, and by $\left[: j_{L, z}^{a} \phi:\right]_{\tilde{\mathcal{R}}}$ a 
linear combination of the operators : $j_{L, z}^{a} \phi$ : that transforms in the representation $\tilde{\mathcal{R}}$. We have shown:

$$
h\left(\left[: j_{L, z}^{a} \phi:\right]_{\tilde{\mathcal{R}}}\right)=\frac{f^{2}}{2} c_{\mathcal{R}}^{(2)}+1+\frac{f^{2}}{2}\left(1-k f^{2}\right)\left(c_{\tilde{\mathcal{R}}}^{(2)}-c_{\mathcal{R}}^{(2)}\right)+\mathcal{O}\left(f^{4}\right) .
$$

The interpretation of this semi-classical result is as follows. At zero Wess-Zumino coupling $k=0$, we find that the conformal dimension at leading order is $f^{2} c_{\tilde{\mathcal{R}}}^{(2)} / 2+1$, namely the quadratic Casimir of the representation in which the total wave-function $j \phi$ transforms times the inverse radius squared, plus one for the fact that we are looking at a descendant state. That is as for a naive evaluation of the conformal dimension of the derivative of an ordinary point-particle wave function in representation $\tilde{\mathcal{R}}$. Note that at the WZW point $k f^{2}=1$, we also recuperate the usual behavior, which is that only the representation of the primary state $\phi$ counts for the basic conformal dimension, while currents add precisely one to the conformal dimension, independent of the representation in which the descendant state transforms. Thus the formula interpolates between these two intuitive behaviors, linearly in $k f^{2}$. Notice that the corrections to the dimension at the WZW point come from the logarithmic term in the current-primary OPE (4.2).

This result illustrates the fact that the bulk partition function will split into a sum over (mini-superspace) representations of the supergroup with conformal dimensions depending on the representation in question. That demonstrates that this behavior, observed in boundary partition functions [9], extends to bulk partition functions. This structure carries over to both left and right conformal dimensions simultaneously.

Indeed, let us turn to the calculation of the anti-holomorphic conformal dimension of the operator : $j_{L, z}^{a} \phi:$. As previously we compute the OPE between the anti-holomorphic stress-tensor and the operator:

$$
\begin{aligned}
\bar{T}(\bar{z}): & j_{L, z}^{a} \phi:(w)=\lim _{: x \rightarrow w:} \bar{T}(\bar{z}) j_{L, z}^{a}(x) \phi(w) \\
= & \lim _{: x \rightarrow w:}\left\{\left(\frac{\bar{\partial} j_{L, z}^{a}(x)}{\bar{z}-\bar{x}}\right) \phi(w)+j_{L, z}^{a}(x)\left(\frac{\bar{\Delta}_{\phi} \phi(w)}{(\bar{z}-\bar{w})^{2}}+\frac{\bar{\partial} \phi(w)}{\bar{z}-\bar{w}}\right)\right\} \\
= & \frac{: \bar{\partial} j_{L, z}^{a} \phi:(w)}{\bar{z}-\bar{w}}+A^{a}{ }_{c} \frac{1}{(\bar{z}-\bar{w})^{2}}: j_{L, z}^{c} \phi:(w) \\
& +\frac{\bar{\Delta}_{\phi}: j_{L, z}^{a} \phi:(w)}{(\bar{z}-\bar{w})^{2}}+\frac{: j_{L, z}^{a} \bar{\partial} \phi:(w)}{\bar{z}-\bar{w}}
\end{aligned}
$$

Hence we have:

$$
\bar{T}(\bar{z}): j_{L, z}^{a} \phi:(w)=\frac{\bar{\Delta}_{\phi}: j_{L, z}^{a} \phi:(w)-\frac{c_{-}}{\left(c_{+}+c_{-}\right)^{2}} i f^{a}{ }_{b c} t^{b}: j_{L, z}^{c} \phi:(w)}{(\bar{z}-\bar{w})^{2}}+\frac{\bar{\partial}: j_{L, z}^{a} \phi:(w)}{\bar{z}-\bar{w}}+\mathcal{O}\left(f^{4}\right) .
$$

That leads to the conformal dimension:

$$
\bar{h}\left(\left[: j_{L, z}^{a} \phi:\right]_{\tilde{\mathcal{R}}}\right)=\frac{f^{2}}{2} c_{\mathcal{R}}^{(2)}+\frac{f^{2}}{2}\left(1-k f^{2}\right)\left(c_{\tilde{\mathcal{R}}}^{(2)}-c_{\mathcal{R}}^{(2)}\right)+\mathcal{O}\left(f^{4}\right) .
$$


This is identical to the previous result, except for the lack of shift by one (since we are acting with the holomorpic component of the left current). Finally one can perform the same computation for the operators : $j_{L, \bar{z}}^{a} \phi:$. One finds:

$$
\begin{aligned}
& h\left(\left[: j_{L, \bar{z}}^{a} \phi:\right]_{\tilde{\mathcal{R}}}\right)=\frac{f^{2}}{2} c_{\mathcal{R}}^{(2)}+\frac{f^{2}}{2}\left(1+k f^{2}\right)\left(c_{\tilde{\mathcal{R}}}^{(2)}-c_{\mathcal{R}}^{(2)}\right)+\mathcal{O}\left(f^{4}\right) \\
& \bar{h}\left(\left[: j_{L, \bar{z}}^{a} \phi:\right]_{\tilde{\mathcal{R}}}\right)=\frac{f^{2}}{2} c_{\mathcal{R}}^{(2)}+1+\frac{f^{2}}{2}\left(1+k f^{2}\right)\left(c_{\tilde{\mathcal{R}}}^{(2)}-c_{\mathcal{R}}^{(2)}\right)+\mathcal{O}\left(f^{4}\right)
\end{aligned}
$$

One can perform similar computations for operators that are composites of a rightcurrent and a primary operator. The conformal dimensions of these operators can also be deduced from the symmetry of the model under the simultaneous interchanges $g \leftrightarrow g^{-1}$ and $z \leftrightarrow \bar{z}$.

A basis of operators. At the Wess-Zumino-Witten points $k f^{2}=1$ the $\bar{z}$-component (respectively $z$-component) of the left current (respectively right current) vanishes, and the left current (respectively right current) is holomorphic (respectively anti-holomorphic). Thus we can expand the currents in a Laurent expansion. The spectrum is generated by acting on the affine primary fields with the modes of the current. It is spanned by the operators:

$$
\left\{j_{-n_{1}}^{a_{1}} j_{-n_{2}}^{a_{2}} \ldots j_{-n_{p}}^{a_{p}} \phi\right\}
$$

where $\phi$ is an affine primary operator and the currents $j^{a_{i}}$ can be either the left-current $J^{a_{i}}$ or the right-current $\bar{J}^{a_{i}}$. In fact, all the negative modes of the currents $J_{-n}^{a}$ (respectively $\bar{J}_{-n}^{a}$ ) can be generated by successive commutations of the first negative mode $J_{-1}^{a}$ (respectively $\left.\bar{J}_{-1}^{a}\right)$. This is most easily seen by working in the Chevalley basis for the generators of the bosonic subalgebra. As a consequence the spectrum is also spanned by the operators:

$$
\left\{j_{-1}^{a_{1}} j_{-1}^{a_{2}} \ldots j_{-1}^{a_{p}} \phi\right\}
$$

Finally, we notice that for any operator $\chi$, the operator $J_{-1}^{a} \chi$ is the regular term in the OPE between the current $J^{a}$ and the operator $\chi$. Thus we can rewrite $J_{-1}^{a} \chi=: J^{a} \chi$ :. So the previous set of operators spanning the spectrum can be rewritten as:

$$
\left\{: j^{a_{1}}: j^{a_{2}} \ldots: j^{a_{p}} \phi: \ldots::\right\} \text {. }
$$

We wrote the spectrum in this unusual form since it has the advantage that these operators are also defined away from the WZW point.

At a generic point of the moduli space both the left- and the right-currents have two non-vanishing components. Since both left and right invariant one-forms generate a basis for the cotangent bundle in spacetime, the sets of operators generated by acting with leftcurrents or with right-currents on primary fields are isomorphic. This indicates that we have two (overcomplete) bases of operators:

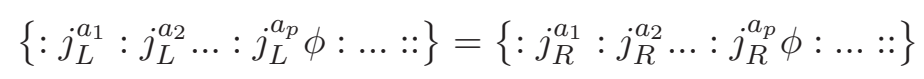

where $\phi$ is a primary field as defined in section 4 , and $j_{L}^{a_{i}}$ (respectively $j_{R}^{a_{i}}$ ) can be either the $z$ - or $\bar{z}$-component of the left current (respectively right current). Of course, a mixture of 
left and right current components is also an allowed choice. We can compute the conformal dimensions of the operators of the sets (6.16) by following the computation given at the beginning of this section. The knowledge of the current-current OPEs (2.5) and of the current-primary OPEs (4.2) up to terms of order $f^{4}$ allows the computation of the conformal dimensions up to terms of order $f^{4}$. Following the logic of section 5 it is then possible to compute order by order in $f^{2}$ the current-current OPEs, the current-primary OPEs and finally the conformal dimensions of the operators (6.16). The recursive calculation may allow for a closed solution.

Let us stress that the spectrum can be generated by acting with the currents on a rather small set of primary operators. The current primaries at any point of the moduli space are in one-to-one correspondence with the affine primaries at the WZW points. In particular the set of current primaries is much smaller than the set of Virasoro primaries. Using the current algebra allows to take advantage of the extension of the symmetry algebra at particular points of the moduli space, namely the WZW points. In other words, in the scheme proposed here, we attempt to maximally exploit the presence of WZW lines in the two-dimensional moduli space of $G_{L} \times G_{R}$ invariant supergroup sigma-models.

\section{The classical and quantum integrability}

The two-dimensional field theory under consideration is classically integrable in the sense that one can code the equations of motion in the demand that a connection depending on a spectral parameter is flat, thus leading to an infinite set of non-local conserved charges. We give the proof of this fact for a generic principal chiral model with Wess-Zumino term in appendix G.

For the model to be quantum integrable, there needs to be an infinite set of conserved charges in the quantum theory. There are circumstances in which anomalies prevent the lifting of the charges from the classical to the quantum theory. It is important to argue that this is not the case for the supergroup sigma-models under consideration here.

Beyond the usual conserved charges $Q_{(0)}^{a}$ associated to the group action(s) on itself, a first set of non-local conserved charges can be defined as [20]:

$$
Q_{(1)}^{a}=N \int d \sigma j_{\sigma}^{a}+\int d \sigma_{1} d \sigma_{2} \epsilon\left(\sigma_{1}-\sigma_{2}\right) f^{a}{ }_{b c} j_{\tau}^{c}\left(\tau, \sigma_{1}\right) j_{\tau}^{b}\left(\tau, \sigma_{2}\right),
$$

where $\tau, \sigma$ are time- and space-coordinates, the factor $N$ is an appropriate normalization constant, and the function $\epsilon$ takes the values \pm 1 depending on the sign of its argument. The non-local charges exists for both left and right currents. The proof of conservation of the non-local charge runs through the fact that the current $j$ is conserved, and the validity of the Maurer-Cartan equation. When both equations are preserved in the quantum theory, the (normal ordered) non-local charges survive (since from the first non-local charges, all others can be generated through commutation with the charges associated to the global symmetries).

It should be clear now that we can view the fixing of higher order terms in the currentcurrent operator products by demanding the vanishing of Maurer-Cartan operator as demanding OPEs compatible with the quantum integrability of the model. Conversely, the 
fact that one can find such OPEs in this model (using the special algebraic properties of the supergroup) lend credence to this hypothesis. It would be useful to make the link between the existence of the Yangian and the form of the current-current operator product expansions even more manifest.

The main threat to the existence of the non-local charge (7.1) comes from the UVdivergence in its definition. In the quadratic term, the current components are both integrated, and the integration involves a region in which the currents come very close to one another, thus necessitating a UV regulator that could potentially render the non-local charges anomalous.

We will now show that in the models at hand, these potential UV divergences are absent, at least to the first few orders in perturbation theory, and presumably to all orders. From the current algebra (2.5), we see that:

$$
f^{a}{ }_{c b} j_{L}^{b}(z) j_{L}^{c}(w)=f^{a}{ }_{c b} j_{L}^{b}(z) j_{L}^{c}(w):+\mathcal{O}\left(f^{4}\right)
$$

which is true for the $z$ and $\bar{z}$ components of the currents. This follows from the fact that the tensors $\kappa^{b c}, A_{d e}^{b c}, B_{d e}^{b c}$ and $C_{d e}^{b c}$ appearing in (2.5) are graded-symmetric in the indices $b, c$. Moreover the double contraction of structure constants (the Killing form) also vanishes. This is a proof of the consistency of the current algebra with quantum integrability to second order. It is a strong suggestion of quantum integrability to all orders, a property which is closely tied to quantum conformal invariance.

\section{Conclusions}

In this paper we continued the investigation of the conformal current algebra in non-linear sigma models on supergroups. The left and right current algebra closes on itself and a primary adjoint operator. The current algebra as well as the current-primary OPEs are tightly constrained by the Maurer-Cartan equation and current conservation, and can be computed order by order in a semi-classical expansion. We argued that one can view the Hilbert space of the theory as generated by currents acting on primaries, since WZW lines exist in the moduli space of theory. We initiated the (perturbative) computation of the spectrum, and argued for the possibility of a recursive bootstrap. We discussed the quantum integrability of the model, and tied it to properties of the current algebra. We hope our analysis contributes to the determination of an explicit solution to the full bulk spectrum of two-dimensional conformal field theories on supergroups and their cosets.

\section{Acknowledgments}

We would like to thank Sujay Ashok, Costas Bachas, Denis Bernard, Vladimir Fateev, Frank Ferrari, Matthias Gaberdiel, Bernard Julia, Anatoly Konechny, Thomas Quella, Sylvain Ribault and Walter Troost for useful questions and helpful discussions. J.T. would like to acknowledge support by ANR grant ANR-09-BLAN-0157-02. The work of R.B. was supported in part by the Belgian Federal Science Policy Office through the Interuniversity Attraction Pole IAP VI/11 and by FWO-Vlaanderen through project G.0428.06. 


\section{A Operator products involving composite operators}

In this appendix we discuss the computation of OPEs involving composite operators. We consider the following OPE:

$$
\lim _{z \rightarrow w} A(z): B C:(w) .
$$

The composite operator : $B C:(w)$ is defined as the term multiplied by $(x-w)^{0}(\bar{x}-\bar{w})^{0}$ in the OPE between the operators $B(x)$ and $C(w)$. To compute the OPE (A.1) we use a point splitting procedure. We denote the extraction of the normal ordered term by the limit : $B C:(w)=\lim _{: x \rightarrow w:} B(x) C(w)$. This symbolizes that at the end of the calculation we take the limit $x \rightarrow w$, and discard all terms that are singular in $x-w$ in this limit.

To compute the operator product of the operator $A$ with the composite operator : $B C$ : we proceed as follows. On the one hand we perform the OPE of the operators $A$ and $B$, and then we perform the OPE of the result with $C$. On the other hand we perform the OPE of the operators $A$ and $C$, and then we perform the OPE of the result with $B$. Eventually take the regular limit $: x \rightarrow w:$ and add up the two terms. Additional details about these operations follow.

- First let us consider the OPE between $A(z)$ and $B(x)$. We evaluate the result at the point $x$ - otherwise taking the regular limit $: x \rightarrow w:$ would become cumbersome. Let us consider one term in the OPE between $A(z)$ and $B(x)$ :

$$
A(z) B(x)=\ldots+(z-x)^{\Delta_{D}-\Delta_{A}-\Delta_{B}}(\bar{z}-\bar{x})^{\bar{\Delta}_{D}-\bar{\Delta}_{A}-\bar{\Delta}_{B}} D(x)+\ldots
$$

where $\Delta_{O}$ (respectively $\bar{\Delta}_{O}$ ) stands for the holomorphic (respectively antiholomorphic) conformal dimension of an operator $O$. For simplicity we consider a term in which no logarithm appears, but the generalization is straightforward. We have to perform the OPE of the right-hand side with the operator $C(w)$. Let us consider one term in the result:

$$
\begin{aligned}
& (z-x)^{\Delta_{D}-\Delta_{A}-\Delta_{B}}(\bar{z}-\bar{x})^{\bar{\Delta}_{D}-\bar{\Delta}_{A}-\bar{\Delta}_{B}} D(x) C(w)= \\
& \quad \ldots+(x-w)^{\Delta_{E}-\Delta_{D}-\Delta_{C}}(\bar{x}-\bar{w})^{\bar{\Delta}_{E}-\bar{\Delta}_{D}-\bar{\Delta}_{C}} \\
& \quad \times(z-x)^{\Delta_{D}-\Delta_{A}-\Delta_{B}}(\bar{z}-\bar{x})^{\bar{\Delta}_{D}-\bar{\Delta}_{A}-\bar{\Delta}_{B}} E(w)+\ldots
\end{aligned}
$$

Now to take the normal ordered limit $: x \rightarrow w:$, we expand the functions depending on $x$ in the neighborhood of $w$, namely, we write:

$$
(z-x)^{\Delta}=(z-w)^{\Delta}-\Delta(x-w)(z-w)^{\Delta-1}+\ldots
$$

and we keep only the terms that end up with no factor of $(x-w)$. The same manipulations have to be done for the anti-holomorphic factors. If both $\Delta_{E}-\Delta_{D}-\Delta_{C}$ and $\bar{\Delta}_{E}-\bar{\Delta}_{D}-\bar{\Delta}_{C}$ are non-positive integers, then the term we isolated in the previous steps contributes to the OPE (A.1) as:

$$
\lim _{z \rightarrow w} A(z): B C:(w)=\ldots+\#(z-w)^{\Delta_{E}-\Delta_{A}-\Delta_{B}-\Delta_{C}}(\bar{z}-\bar{w})^{\bar{\Delta}_{E}-\bar{\Delta}_{A}-\bar{\Delta}_{B}-\bar{\Delta}_{C}} E(w)
$$


with numerical coefficient:

$$
\begin{aligned}
\#=( & -1)^{-\Delta_{E}+\Delta_{D}+\Delta_{C}}(-1)^{-\bar{\Delta}_{E}+\bar{\Delta}_{D}+\bar{\Delta}_{C}} \\
& \times \frac{\left(\Delta_{D}-\Delta_{A}-\Delta_{B}\right)\left(\Delta_{D}-\Delta_{A}-\Delta_{B}-1\right) \ldots\left(\Delta_{E}-\Delta_{A}-\Delta_{B}-\Delta_{C}+1\right)}{\left(-\Delta_{E}+\Delta_{D}+\Delta_{C}\right) !} \\
& \times \frac{\left(\bar{\Delta}_{D}-\bar{\Delta}_{A}-\bar{\Delta}_{B}\right)\left(\bar{\Delta}_{D}-\bar{\Delta}_{A}-\bar{\Delta}_{B}-1\right) \ldots\left(\bar{\Delta}_{E}-\bar{\Delta}_{A}-\bar{\Delta}_{B}-\bar{\Delta}_{C}+1\right)}{\left(-\bar{\Delta}_{E}+\bar{\Delta}_{D}+\bar{\Delta}_{C}\right) !} .
\end{aligned}
$$

Let us stress that a given term in the result of the OPE (A.1) may receive contributions from an infinite number of terms in the OPE between $A$ and $B$. This makes the computation of OPEs involving composite operators rather involved. One may need to resort to perturbation theory in a small parameter to render the calculation manageable. The perturbation theory that we use in the bulk of the paper is explained in section 5 and in the appendices B and C.

- Let us turn to the OPE between $A(z)$ and $C(w)$, which we evaluate at the point $w$. This second step is simpler than the first. Again, we concentrate on one term in this OPE:

$$
A(z) C(w)=\ldots+(z-w)^{\Delta_{F}-\Delta_{A}-\Delta_{B}}(\bar{z}-\bar{w})^{\bar{\Delta}_{F}-\bar{\Delta}_{A}-\bar{\Delta}_{B}} F(w)+\ldots
$$

We then have to perform the OPE between the right-hand side and the operator $B(x)$. We evaluate the result at the point $w$. Let us write down one term in the result:

$$
\begin{aligned}
& (z-w)^{\Delta_{F}-\Delta_{A}-\Delta_{B}}(\bar{z}-\bar{w})^{\bar{\Delta}_{F}-\bar{\Delta}_{A}-\bar{\Delta}_{B}} B(x) F(w)= \\
& \ldots+(z-w)^{\Delta_{F}-\Delta_{A}-\Delta_{B}}(\bar{z}-\bar{w})^{\bar{\Delta}_{F}-\bar{\Delta}_{A}-\bar{\Delta}_{B}} \\
& \times(x-w)^{\Delta_{G}-\Delta_{B}-\Delta_{F}}(\bar{x}-\bar{w})^{\bar{\Delta}_{G}-\bar{\Delta}_{B}-\bar{\Delta}_{F}} G(w)+\ldots
\end{aligned}
$$

Finally we take the straightforward normal ordered limit $: x \rightarrow w:$, that discards all the terms except for the one with $\Delta_{G}-\Delta_{B}-\Delta_{F}=\bar{\Delta}_{G}-\bar{\Delta}_{B}-\bar{\Delta}_{F}=0$. Thus only the regular term : $B F:(w)$ in the OPE between $B(x)$ and $F(w)$ survives. We obtain the following contribution to the OPE (A.1):

$$
\lim _{z \rightarrow w} A(z): B C:(w)=\ldots+(z-w)^{\Delta_{F}+\Delta_{B}-\Delta_{A}-\Delta_{C}}(\bar{z}-\bar{w})^{\bar{\Delta}_{F}+\bar{\Delta}_{B}-\bar{\Delta}_{A}-\bar{\Delta}_{C}}: B F:(w) .
$$

Simplification in the case of a holomorphic operator. The computation of the singular terms in the OPE (A.1) simplifies if the operator $A(z)$ is holomorphic. Let us consider a term of the form (A.2). Since the operator $A$ is holomorphic there is no dependence on $\bar{z}$, so $\bar{\Delta}_{D}-\bar{\Delta}_{A}-\bar{\Delta}_{B}=0$. Let us also assume that $\Delta_{D}-\Delta_{A}-\Delta_{B}$ is an integer. The question is whether such a term may contribute to a pole in the OPE (A.1), i.e. a term of the form (A.4) with $\Delta_{E}-\Delta_{A}-\Delta_{B}-\Delta_{C}$ a negative integer (and $\bar{\Delta}_{E}-\bar{\Delta}_{A}-\bar{\Delta}_{B}-\bar{\Delta}_{C}=0$ ). But this is only possible if $\Delta_{D}-\Delta_{A}-\Delta_{B}$ is already a negative integer, since otherwise the coefficient (A.5) vanishes.

It follows from the previous discussion that under the assumption that only integer powers of $(z-x)$ appear in the OPE between the operators $A(z)$ and $B(x)$, then in the 
computation of singular terms in the OPE (A.1) one can truncate the OPE between $A(z)$ and $B(x)$ to the singular terms only (i.e. keep only the poles in $(z-x)$ ). That specific feature of this special case is put to good use in some standard calculations in two-dimensional conformal field theory [17].

\section{B The semi-classical behavior of the OPE coefficients}

At large radius, namely in the limit $f^{2} \rightarrow 0$ (either at fixed level $k$ or at fixed $k f^{2}$ ), the target space flattens and the worldsheet theory becomes free. More precisely we obtain a theory of $d$ free bosons, where $d$ is the dimension of the adjoint representation of the super Lie algebra. Among these bosons, some are commuting and some are anti-commuting, depending on whether they can be associated to bosonic or fermionic coordinates of target space. At fixed $k f^{2}$ the $f^{2} \rightarrow 0$ limit is the semi-classical limit of the model.

Our goal in this appendix is to evaluate the behavior at large radius (small $f^{2}$ ) of the terms appearing in the current-current and current-primary OPEs. Let us start with the action of the model:

$$
\begin{aligned}
S & =S_{k i n}+S_{W Z} \\
S_{k i n} & =\frac{1}{16 \pi f^{2}} \int d^{2} x T r^{\prime}\left[-\partial^{\mu} g^{-1} \partial_{\mu} g\right] \\
S_{W Z} & =-\frac{i k}{24 \pi} \int_{B} d^{3} y \epsilon^{\alpha \beta \gamma} \operatorname{Tr}^{\prime}\left(g^{-1} \partial_{\alpha} g g^{-1} \partial_{\beta} g g^{-1} \partial_{\gamma} g\right) .
\end{aligned}
$$

We write the group element as:

$$
g=e^{f X}=e^{i f X_{a} t^{a}}
$$

where the $X_{a}$ are coordinates on the supergroup and the matrices $t^{a}$ are the generators of the Lie superalgebra. The kinetic term and the Wess-Zumino term become:

$$
\begin{aligned}
S_{k i n} & =\frac{1}{4 \pi} \int d^{2} z\left(\partial X_{a} \bar{\partial} X^{a}-\frac{f^{2}}{12} f_{f e}^{a} f_{a c b} X^{b} \partial X^{c} X^{e} \bar{\partial} X^{f}+\ldots\right) \\
S_{W Z} & =-\frac{k f^{2}}{12 \pi} \int d^{2} z\left(f f_{a b c} X^{c} \partial X^{b} \bar{\partial} X^{a}+\ldots\right) .
\end{aligned}
$$

Written in this way the theory describes a set of interacting bosons (some of which are anti-commuting). The quadratic terms in the action give rise to the free propagator:

$$
X^{a}(z, \bar{z}) X^{b}(w, \bar{w})=-\kappa^{a b} \log \mu^{2}|z-w|^{2},
$$

where $\mu$ is an infrared regulator. The propagator behaves like $\mathcal{O}\left(f^{0}\right)$, whereas a vertex with $p+2$ legs (i.e. Lie algebra indices) behaves as $\mathcal{O}\left(f^{p}\right)$. It follows that the theory reduces to a theory of free bosons in the semi-classical limit, as anticipated. At fixed $k f^{2}$ and for each interaction vertex, the power of the coupling constant $f$ is equal to the number of structure constants that appear. Since we are interested in computing OPEs involving the 
currents and the primary fields, let us write these fields in terms of the bosons $X^{a}$ :

$$
\begin{aligned}
\frac{j_{L, z}^{a}}{c_{+}} & =\left(\partial g g^{-1}\right)^{a}=i\left(f \partial X^{a}+f^{2} \frac{f_{b c}^{a}}{2} X^{c} \partial X^{b}+\frac{f^{3}}{6} f_{b c}^{a} f_{d e}^{c} \partial X^{e} X^{d} X^{b}+\ldots\right) \\
\frac{j_{L, \bar{z}}^{a}}{c_{-}} & =\left(\bar{\partial} g g^{-1}\right)^{a}=i\left(f \bar{\partial} X^{a}+f^{2} \frac{f^{a}{ }_{c b}}{2} X^{b} \bar{\partial} X^{c}+\frac{f^{3}}{6} f_{b c}^{a} f_{d e}^{c} \bar{\partial} X^{e} X^{d} X^{b}+\ldots\right), \\
\phi & =e^{i f X_{a} t^{a}}=i f X_{a} t^{a}-f^{2} X_{a} t^{a} X_{b} t^{b}+\ldots
\end{aligned}
$$

where in the last line the generators $t^{a}$ are taken in the representation associated to the primary field $\phi$.

The semi-classical behavior of the current-current OPE. We study the semiclassical behavior of the OPE between two $z$-components of the left-current. The discussion generalizes straightforwardly to other current-current OPEs. We assume that the only operators that appear in the result of this OPE are composites of (derivatives of) left currents. This is true at the WZW point, and can presumably be proven at any point using conformal perturbation theory. Let us isolate one term in this OPE:

$$
j_{L, z}^{a}(z) j_{L, z}^{b}(w)=\ldots+A^{a b}{ }_{a_{p} a_{p-1} \ldots a_{2} a_{1}}(z-w, \bar{z}-\bar{w}): j_{L, z}^{a_{1}}: j_{L, z}^{a_{2}} \ldots: j_{L, z}^{a_{p-1}} j_{L, z}^{a_{p}}: \ldots::(w)+\ldots
$$

Our goal is to evaluate the behavior of the tensor $A^{a b}{ }_{a_{p} \ldots a_{1}}(z-w, \bar{z}-\bar{w})$ when the parameter $f$ is small. The reasoning will not depend on the particular current component, nor on the presence of further derivative operators.

To proceed we use the expression (B.5) of the currents in terms of the bosonic fields $X^{a}$. First let us focus on the leading term in the expansion (B.5). We consider the OPE:

$$
\begin{aligned}
\partial X^{a}(z) \partial X^{b}(w)= & \ldots+\tilde{A}^{a b}{ }_{a_{p} a_{p-1} \ldots a_{2} a_{1}}(z-w, \bar{z}-\bar{w}): \\
& \partial X^{a_{1}}: \partial X^{a_{2}} \ldots: \partial X^{a_{p-1}} \partial X^{a_{p}}: \ldots::(w)+\ldots
\end{aligned}
$$

The behavior of the tensor $\tilde{A}^{a b}{ }_{a_{p} a_{p-1} \ldots a_{2} a_{1}}$ as a function of the parameter $f$ will give the behavior of the tensor $A^{a b}{ }_{a_{p} \ldots a_{1}}(z-w, \bar{z}-\bar{w})$ defined in equation (B.7). As a first step let us consider the following three-point function:

$$
\left\langle\partial X^{a}(z) \partial X^{b}(w): \partial X^{a_{1}}: \partial X^{a_{2}} \ldots: \partial X^{a_{p-1}} \partial X^{a_{p}}: \ldots::(x)\right\rangle_{\text {connected }}
$$

We consider only the contribution of connected Feynman diagrams to this correlation function. Indeed, if the external operators $\partial X^{a}(z)$ and $\partial X^{b}(w)$ are contracted on different pieces of a disconnected Feynman diagram, then the result contributes to the regular term : $\partial X^{a}(x) \partial X^{b}(w)$ : on the right-hand side of the OPE (B.8). Thus to compute the nontrivial terms in this OPE one needs to consider only the Feynman diagrams for which the external operators $\partial X^{a}(z)$ and $\partial X^{b}(w)$ are connected. But this in turn implies that the Feynman diagram is fully connected. Indeed, if this were not the case then one connected piece of the Feynman diagram has for external lines operators coming from the composite operator : $\partial X^{a_{1}}: \partial X^{a_{2}} \ldots: \partial X^{a_{p-1}} \partial X^{a_{p}}: \ldots::(x)$ only. Such a piece would depend on the coordinate $x$ only, and would necessarily be zero by translation invariance. This shows that we need to consider only fully connected Feynman diagrams. 
Now let us evaluate the $f$-dependence of a connected Feynman diagram contributing to (B.9). We will show by induction the following statement: a connected Feynman diagram in the theory (B.3) with $p+2$ external legs behaves like $\mathcal{O}\left(f^{p}\right)$. This is the case for $p=0$ since the propagator is of order $f^{0}$. Now let us assume that the statement has been proven for $p<n+2$, and consider a Feynman diagram with $n+2$ external lines. We isolate $m$ of these external legs that are contracted on the same vertex with $m+1$ legs. This piece is of order $f^{m-1}$. The other piece of the Feynman diagram has $n+2-m+1$ external lines, and by induction is of order $f^{n-m+1}$. Thus the result is of order $f^{n}$, and the proof is completed. We deduce that:

$$
\left\langle\partial X^{a}(z) \partial X^{b}(w): \partial X^{a_{1}}: \partial X^{a_{2}} \ldots: \partial X^{a_{p-1}} \partial X^{a_{p}}: \ldots::(x)\right\rangle_{\text {connected }}=\mathcal{O}\left(f^{p}\right) .
$$

Since two-point functions of (composites of) the fields $X^{a}$ behave at least as $\mathcal{O}\left(f^{0}\right)$, we can now combine the previous result with equation (B.5) to evaluate the order of the term in the current OPE under consideration: ${ }^{4}$

$$
\frac{j_{L, z}^{a}(z)}{f c_{+}} \frac{j_{L, z}^{b}(w)}{f c_{+}}=\ldots+\mathcal{O}\left(f^{p}\right): \frac{j_{L, z}^{a_{1}}}{f c_{+}}: \frac{j_{L, z}^{a_{2}}}{f c_{+}} \ldots: \frac{j_{L, z}^{a_{p-1}}}{f c_{+}} \frac{j_{L, z}^{a_{p}}}{f c_{+}}: \ldots::(w)+\ldots
$$

Given that $f c_{+}=\mathcal{O}\left(f^{-1}\right)$, we obtain:

$$
j_{L, z}^{a}(z) j_{L, z}^{b}(w)=\ldots+\mathcal{O}\left(f^{2 p-2}\right): j_{L, z}^{a_{1}}: j_{L, z}^{a_{2}} \ldots: j_{L, z}^{a_{p-1}} j_{L, z}^{a_{p}}: \ldots::(w)+\ldots
$$

This is a property we repeatedly confirm as well as use in the bulk of the paper.

The semi-classical behavior of the current-primary OPE. We can perform a similar analysis to determine the behavior of the terms in the current-primary OPE at large radius. Let us consider a primary field $\phi$. We assume that all the terms that appear in the OPE between a left current and this primary field are composite operators including an arbitrary number of left currents and one field $\phi$ only. This is the case at the WZW point. Then by continuously deforming the OPEs away from the WZW point, this is the case over the whole moduli space of the theory. Let us isolate one term in the OPE between the left current $j_{L, z}^{a}$ and the primary field $\phi$ :

$$
j_{L, z}^{a}(z) \phi(w)=\ldots+B^{a}{ }_{a_{p} a_{p-1} \ldots a_{1}}(z-w, \bar{z}-\bar{w}): j_{L, z}^{a_{1}}: j_{L, z}^{a_{2}} \ldots: j_{L, z}^{a_{p}} \phi: \ldots::(w)+\ldots
$$

Our goal is to evaluate the behavior of the tensor $B^{a}{ }_{a_{p} \ldots a_{1}}(z-w, \bar{z}-\bar{w})$ when the parameter $f^{2}$ is small. The composite operator we wrote down does not have any derivative and contains only $z$-components of the left current, but the result would be the same for a more general operator. Only the number $p$ of currents will be relevant. Following the previous reasoning one can show that:

$$
\left\langle\partial X^{a}(z) X^{b}(w): \partial X^{a_{1}}: \partial X^{a_{2}} \ldots: \partial X^{a_{p}} X^{a_{p+1}}: \ldots::(x)\right\rangle_{\text {connected }}=\mathcal{O}\left(f^{p+1}\right) .
$$

\footnotetext{
${ }^{4}$ Using similar methods it can be shown that the subleading terms in equation (B.5) do not modify this conclusion.
} 
Combining this result together with equations (B.5) and (B.6) and the fact that two-points functions are of order $\mathcal{O}\left(f^{0}\right)$ we get:

$$
\frac{j_{L, z}^{a}(z)}{f c_{+}} \frac{\phi(w)}{i f}=\ldots+\mathcal{O}\left(f^{p+1}\right): \frac{j_{L, z}^{a_{1}}}{f c_{+}}: \frac{j_{L, z}^{a_{2}}}{f c_{+}} \ldots: \frac{j_{L, z}^{a_{p}}}{f c_{+}} \frac{\phi}{i f}: \ldots::(w)+\ldots
$$

which we rewrite as:

$$
j_{L, z}^{a}(z) \phi(w)=\ldots+\mathcal{O}\left(f^{2 p}\right): j_{L, z}^{a_{1}}: j_{L, z}^{a_{2}} \ldots: j_{L, z}^{a_{p}} \phi: \ldots::(w)+\ldots
$$

This result on the order of magnitude of the operator product is confirmed and used in the bulk of the paper.

\section{Consistency of perturbation theory}

Current-current OPE. In section 5 we explained how to compute the current-current OPEs order by order in a semi-classical expansion. The idea is to ask for the vanishing of the OPE between a current and both current conservation and the Maurer-Cartan equation, order by order in $f^{2}$. These two constraints can be combined as:

$$
j_{L}^{a}(z)\left(\bar{\partial} j_{L, z}^{b}(w)-i f^{2} f_{c d}^{b}: j_{L, z}^{d} j_{L, \bar{z}}^{c}:(w)\right)=0 .
$$

For this perturbative method to be consistent a term of order $f^{2 n}$ in the current-current OPEs should not spoil the vanishing of the previous OPE up to order $f^{2 n-2}$. The subtlety lies in the computation of the OPE involving the composite operator in equation (C.1). Indeed the fact that the leading singularity in the current-current OPE has a coefficient of order $f^{-2}$ threatens to generates terms of low order in $f^{2}$ in this computation. In this appendix we will show that a term of order $f^{2 n}$ in the current-current OPE does produce terms of order $f^{2 n}$ in the OPE between a current and the composite operator appearing in equation (C.1), namely $f^{2} f_{c d}^{b}: j_{L, z}^{d} j_{L, \bar{z}}^{c}:$

As a preliminary step let us prove the following useful lemma. We consider a composite of $p$ currents $: j: j: j \ldots j: \ldots::$ that we write symbolically $: j^{p}:$. Then the OPE of this operator with one current $j$ is at most of order $f^{-2}$ :

$$
j(z): j^{p}:(w)=\mathcal{O}\left(f^{-2}\right) .
$$

To prove this property we rewrite the current in terms of the bosons $X^{a}$ using equation (B.5). Schematically we have:

$$
j=f^{-2} \sum_{n=0}^{\infty} \# f^{n+1}: X^{n+1}:
$$

where we kept the numerical factors, possible derivatives acting on the fields $X$, and the index structures implicit to simplify the formula. Similarly the composite operator $: j^{p}:$ is written as:

$$
: j^{p}:=f^{-2 p} \sum_{m=0}^{\infty} \# f^{m+p}: X^{m+p}:
$$


To evaluate the OPE between the current $j$ and the composite operator $: j^{p}:$ we need to evaluate the OPE between operators of the form : $X^{q}:$. Remember that the propagator for the field $X$ is of order $f^{0}$, and that the $n$-point vertex is of order $f^{n-2}$. We deduce:

$$
: X^{q_{1}}:(z): X^{q_{2}}:(w)=\sum_{q=0}^{\infty} \mathcal{O}\left(f^{\left|q_{1}-q_{2}\right|-q}\right): X^{q}:(z)
$$

In the previous equation the estimation of the order of the terms is rough (especially for large $q$ ) but it will be sufficient for our purposes. The proof is similar to the argument given below (B.9) (except that in the present case disconnected Feynman diagrams contribute). We deduce an estimation for the order of the terms in the OPE (C.2)

$$
j(z): j^{p}:(w)=f^{-2 p-2} \sum_{n, m=0}^{\infty} f^{n+m+p+1} \sum_{q=0}^{\infty} \mathcal{O}\left(f^{|n+1-m-p|-q}\right): X^{q}:
$$

The operators that appear in the OPE (C.2) are themselves (composites of) currents. Let us evaluate the coefficient of a composite operator of the form $: j^{r}:$. According to equation (B.5) the leading-order term in this composite operator written in terms of $X$ 's is:

$$
: j^{r}:=f^{-r}: X^{r}:+\mathcal{O}\left(: X^{r+1}:\right) \text {. }
$$

So to get the order of the coefficient that multiplies and operator $: j^{r}:$, it is enough to look for the coefficient of the terms multiplying $f^{-r}: X^{r}$ : in the OPE (C.6). These terms have a coefficient of order:

$$
f^{-2 p-2+n+m+p+1+|n+1-m-p|}= \begin{cases}f^{2(n+1-p)-2} & \text { if } n+1 \geq m+p \\ f^{2 m-2} & \text { if } n+1 \leq m+p .\end{cases}
$$

Thus this coefficient is of order $\mathcal{O}\left(f^{-2}\right)$. This completes the proof of (C.2).

Now let us come back to the evaluation of the OPE between a current and the composite operator in equation (C.1):

$$
j_{L, z}^{a}(z) i f^{2} f_{c d}^{b}: j_{L, z}^{d} j_{L, \bar{z}}^{c}:(w)
$$

Let us consider one term of order $f^{2 n}$ in the OPE between the operators $j_{L, z}^{a}$ and $j_{L, z}^{d}$, that we write schematically $f^{2 n}: j^{p}:$. To complete the computation we have to perform the OPE of this operator with the remaining current $j_{L, \bar{z}}^{c}$. According to the previous lemma, this OPE produces terms with coefficients of order $f^{-2}$. So we have proven that terms of order $f^{2 n}$ in the current-current OPE produce in the OPE (C.1) terms of order $f^{2+2 n-2}=f^{2 n}$. This proves the consistency of the algorithm to compute the current-current OPE order by order in $f^{2}$.

Current-primary OPE. As explained in section 5 the same logic allows us to perturbatively compute the operator product expansion between a current and a primary operator. The Maurer-Cartan equation can be combined with current conservation to give the constraint:

$$
\phi(z)\left(\bar{\partial} j_{L, z}^{b}(w)+i f^{2} f_{c d}^{b}: j_{L, z}^{d} j_{L, \bar{z}}^{c}:(w)\right)=0
$$


This allows the computation of the $j_{L, z}^{a} . \phi$ OPE order by order in $f^{2}$. The consistency of this algorithm is ensured by a slight generalization of lemma (C.2), namely:

$$
j(z): j^{p} \phi:(w)=\mathcal{O}\left(f^{-2}\right) .
$$

The proof is similar to the proof of formula (C.2).

\section{Conformal current algebra: precisions}

In this appendix we gather various technical results related to the current algebra (2.5).

\section{D.1 The current algebra at order $f^{2}$}

In [4] the current algebra (2.5) was computed at the order of the poles. The discussion of section 5 shows that we can compute the less-singular terms by demanding consistency with current conservation and the Maurer-Cartan equation. In this appendix we will give details of this computation, and derive in particular the value of the new coefficients (2.9) in the current algebra (2.5).

In this particular calculation, we show how to restore various signs that are associated to the fact that we deal with a super Lie algebra. Since we use the special algebraic structure of supergroups with zero Killing form, these signs are crucial. To set up the problem, we establish conventions for the metric inverse and the contraction of indices:

$$
\begin{aligned}
\kappa_{a b} \kappa^{c b} & =\delta_{a}{ }^{c} \\
j_{a} & =\kappa_{a b} j^{b} \\
{\left[t_{a}, t_{b}\right] } & =i t_{c} f^{c}{ }_{a b} .
\end{aligned}
$$

We contract indices south-west north-east. ${ }^{5}$

As explained in section 5 current conservation implies that the tensors $A, B, C$ that appear in each one of the three OPEs (2.5) are equal. To compute them we ask for the vanishing of the OPE between a current and the Maurer-Cartan operator:

$$
c_{-} \partial_{\bar{z}} j_{L, z}^{c}-c_{+} \partial_{z} j_{L, \bar{z}}^{c}-i f_{d e}^{c}: j_{L, z}^{e} j_{L, \bar{z}}^{d}: .
$$

Below we compute the OPE between the (left) current $j_{\bar{z}}^{a}$ and the Maurer-Cartan operator. For ease of writing, we will separate various terms in the calculation. We first calculate the operator product of the current with the first term:

$$
\begin{aligned}
\text { Term } 1= & j_{\bar{z}}^{a}(z) \cdot c_{-} \partial_{\bar{w}} j_{z}^{c}(w) \\
\sim & c_{-} \partial_{\bar{w}}\left(\tilde{c} \kappa^{a c} 2 \pi \delta(z-w)+f_{g}^{a c}\left(\frac{c_{4}-g}{\bar{z}-\bar{w}} j_{z}^{g}(z)+\frac{\left(c_{2}-g\right)}{z-w} j_{\bar{z}}^{g}(z)\right.\right. \\
& \left.+\frac{g}{4} \log |z-w|^{2}\left(\partial_{z} j_{\bar{z}}^{g}(z)-\partial_{\bar{z}} j_{z}^{g}(z)\right)\right)+(-1)^{a c}: j_{z}^{c} j_{\bar{z}}^{a}:(z) \\
& +(A)^{a c}{ }_{g h} \frac{\bar{z}-\bar{w}}{z-w}: j_{\bar{z}}^{g} j_{\bar{z}}^{h}:(z)-(B)^{a c}{ }_{g h} \log |z-w|^{2}: j_{z}^{g} j_{\bar{z}}^{h}:(z) \\
& \left.+(C)^{a c}{ }_{g h} \frac{z-w}{\bar{z}-\bar{w}}: j_{z}^{g} j_{z}^{h}:(z)+\ldots\right)
\end{aligned}
$$

\footnotetext{
${ }^{5}$ These conventions differ only slightly from those in [4].
} 
The second term we take into account comes from contracting the current with the second term in the Maurer-Cartan operator:

$$
\begin{aligned}
\operatorname{Term} 2= & j_{\bar{z}}^{a}(z) \cdot(-) c_{+} \partial_{w} j_{\bar{z}}^{c}(w) \\
\sim & -c_{+} \partial_{w}\left(c_{3} \kappa^{a c} \frac{1}{(\bar{z}-\bar{w})^{2}}+f_{g}^{a c}\left(\frac{c_{4}}{\bar{z}-\bar{w}} j_{\bar{z}}^{g}(w)+\frac{\left(c_{4}-g\right)(z-w)}{(\bar{z}-\bar{w})^{2}} j_{z}^{g}(w)\right.\right. \\
& \left.+\frac{g}{4} \frac{z-w}{\bar{z}-\bar{w}}\left(\partial_{z} j_{\bar{z}}^{g}(w)-\partial_{\bar{z}} j_{z}^{g}(w)\right)+\frac{c_{4}}{2} \partial_{\bar{z}} j_{\bar{z}}^{g}(w)+\frac{c_{4}-g}{2} \frac{(z-w)^{2}}{(\bar{z}-\bar{w})^{2}} \partial_{z} j_{z}^{g}(w)\right) \\
& +: j_{\bar{z}}^{a} j_{\bar{z}}^{c}:(w)+\left(-(A)^{a c}{ }_{g h} \log |z-w|^{2}: j_{\bar{z}}^{g} j_{\bar{z}}^{h}:+(B)^{a c}{ }_{g h} \frac{z-w}{\bar{z}-\bar{w}}: j_{z}^{g} j_{\bar{z}}^{h}:\right. \\
& \left.\left.+(C)^{a c}{ }_{g h} \frac{(z-w)^{2}}{(\bar{z}-\bar{w})^{2}}: j_{z}^{g} j_{z}^{h}:(w)\right)\right)+\ldots
\end{aligned}
$$

Furthermore we have the contractions with the composite piece of the Maurer-Cartan operator. Following appendix A we use a point-splitting procedure and write $f_{d e}^{c}: j_{z}^{e} j_{\bar{z}}^{d}$ : $(w)=\lim _{: x \rightarrow w:} f_{d e}^{c} j_{z}^{e}(x) j_{\bar{z}}^{d}(w)$. Then we distinguish two terms. The simplest is the term where we contract the current component $j_{\bar{z}}^{a}$ with the part at $w$ of the split operator. We then still need to contract further while eliminating singularities as $x$ goes to $w$, but this is easily done: only regular terms survive. We obtain:

$$
\begin{aligned}
\text { Term } 3= & (-i)(-1)^{e a} f_{d e}^{c}\left(\left(c_{3} \kappa^{a d} \frac{1}{(\bar{z}-\bar{w}}\right)^{2} j_{z}^{e}(w)\right. \\
& \left.+f_{g}^{a d}\left(\frac{c_{4}}{\bar{z}-\bar{w}}: j_{z}^{e} j_{\bar{z}}^{g}:(w)+\frac{\left(c_{4}-g\right)(z-w)}{(\bar{z}-\bar{w})^{2}}: j_{z}^{e} j_{z}^{g}:(w)\right)\right) \\
& + \text { order zero in the separation. }
\end{aligned}
$$

There is also the more involved term where we contract first with $j_{z}^{e}(x)$, and then further with $j_{\bar{z}}^{d}(w)$ :

$$
\text { Term } 4=\lim _{: x \rightarrow w:} X^{a e}(z, x)(-i) f_{d e}^{c} j_{\bar{z}}^{d}(w)
$$

where

$$
\begin{aligned}
X^{a e}(z, x) \sim & \tilde{c} \kappa^{a e} 2 \pi \delta(z-x) \\
& +f_{g}^{a e}\left(\frac{c_{4}-g}{\bar{z}-\bar{x}} j_{z}^{g}(z)+\frac{\left(c_{2}-g\right)}{z-x} j_{\bar{z}}^{g}(z)+\frac{g}{4} \log |z-x|^{2}\left(\partial_{z} j_{\bar{z}}^{g}(z)-\partial_{\bar{z}} j_{z}^{g}(z)\right)\right) \\
& +(-1)^{a e}: j_{z}^{e} j_{\bar{z}}^{a}:(z)+A^{a c}{ }_{g h} \frac{\bar{z}-\bar{x}}{z-x}: j_{\bar{z}}^{g} j_{\bar{z}}^{h}:(z)-B^{a c}{ }_{g h} \log |z-x|^{2}: j_{z}^{g} j_{\bar{z}}^{h}:(z) \\
& +C^{a c}{ }_{g h} \frac{z-x}{\bar{z}-\bar{x}}: j_{z}^{g} j_{z}^{h}:(z) \\
& + \text { order } 1 \text { in the separation and higher order in the parameter } f^{2} .
\end{aligned}
$$

Let's sum these four terms and discuss the vanishing of the total operator product order by order. The contact terms and double pole terms were already treated in [4]. We cancel them as follows: 
1. There are terms proportional to $\partial_{\bar{w}} 2 \pi \delta(z-w)$. These have coefficients:

$$
c_{-} \tilde{c} \kappa^{a c}+c_{+} c_{3} \kappa^{a c}
$$

which vanishes since the coefficients of the current algebra (2.6) satisfy:

$$
c_{-} \tilde{c}=-c_{+} c_{3}
$$

2. There are terms proportional to $2 \pi \delta(z-w)$ with coefficient:

$$
\begin{aligned}
& -c_{-} f^{a c}{ }_{g}\left(c_{2}-g\right) j_{\bar{z}}^{g}(w)+c_{+} f^{a c}{ }_{g} c_{4} j_{\bar{z}}^{g}(w)-i f_{d e}^{c} \tilde{c} \kappa^{a e} j_{\bar{z}}^{d}(w) \\
& \quad=-c_{-} f_{g}^{a c}\left(c_{2}-g\right) j_{\bar{z}}^{g}(w)+c_{+} f_{g}^{a c} c_{4} j_{\bar{z}}^{g}(w)-i(-1)^{a}(-1)^{a} f^{a c}{ }_{g} \tilde{c} j_{\bar{z}}^{g}(w)
\end{aligned}
$$

which also vanishes thanks to the relation:

$$
-c_{-}\left(c_{2}-g\right)+c_{+} c_{4}-i \tilde{c}=0 .
$$

3. There are terms proportional to $1 /(\bar{z}-\bar{w})^{2}$ with coefficients:

$$
c_{-}\left(c_{4}-g\right) f_{g}^{a c} j_{z}^{g}+c_{+}\left(c_{4}-g\right) f_{g}^{a c} j_{z}^{g}-i(-1)^{e a} f^{c}{ }_{d e} c_{3} \kappa^{a d} j_{z}^{e}-i f^{c}{ }_{d e} f^{a e}{ }_{g}\left(c_{4}-g\right)^{2} f^{g d}{ }_{h} j_{z}^{h}
$$

where the last term arises from expanding $1 /(z-x)$ and taking into account the further contraction in Term 4. This last term vanishes thanks to the super-Jacobi identity combined with the vanishing of the Killing form. Note that this implies that the second line in Term 4 does not contribute when the contraction between $j_{z}^{g}$ and $j_{\bar{z}}^{d}$ gives rise to either a metric or structure constant. Thus, it can potentially contribute starting at order zero in the separation only. The coefficient of the terms under consideration then vanishes since the coefficient (2.6) satisfies the relation:

$$
\left(c_{-}+c_{+}\right)\left(c_{4}-g\right)+i c_{3}=0 .
$$

4. We now turn to the calculation which is new compared to [4]. In the operator product expansion the simple pole in $1 /(\bar{z}-\bar{w})$ comes with the coefficient:

$$
\begin{aligned}
& c_{-} f_{g}^{a c}\left(c_{4}-g\right) \partial_{\bar{z}} j_{z}^{g}(w)-c_{+} f_{g}^{a c} c_{4} \partial_{z} j_{\bar{z}}^{g}(w) \\
& \quad-c_{-} \frac{g}{4} f^{a c}{ }_{g}\left(\partial_{z} j_{\bar{z}}^{g}-\partial_{\bar{z}} j_{z}^{g}\right)+c_{-} B^{a c}{ }_{g h}: j_{z}^{g} j_{\bar{z}}^{h}:(w) \\
& \quad+c_{+} \frac{g}{4} f^{a c}{ }_{g}\left(\partial_{z} j_{\bar{z}}^{g}-\partial_{\bar{z}} j_{z}^{g}\right)+c_{+} B^{a c}{ }_{g h}: j_{z}^{g} j_{\bar{z}}^{h}:(w) \\
& \quad-i(-1)^{e a} f_{d e}^{c} f_{g}^{a d} c_{4}: j_{z}^{e} j_{\bar{z}}^{g}:(w) \\
& \quad-i f_{d e}^{c} f_{g}^{a e}\left(c_{4}-g\right): j_{z}^{g} j_{\bar{z}}^{d} \\
& \quad-i f_{d e}^{c} f_{g}^{a e}\left(c_{2}-g\right) B^{g d}{ }_{x y}: j_{z}^{x} j_{\bar{z}}^{y}: \\
& \quad+\mathcal{O}\left(f^{2}\right)
\end{aligned}
$$


We use current conservation and the Maurer-Cartan equation to write:

$$
\begin{aligned}
+ & \left(c_{4}-\frac{g}{2}\right) f^{a c}{ }_{g} f_{d e}^{g}: j_{z}^{e} j_{\bar{z}}^{d}:+c_{-} B^{a c}{ }_{e d}: j_{z}^{e} j_{\bar{z}}^{d}:(w) \\
& +c_{+} B^{a c}{ }_{e d}: j_{z}^{e} j_{\bar{z}}^{d}:(w) \\
& +\left(c_{4}-g / 2\right)\left(i f_{e g}^{c} f_{d}^{a g}{ }_{d}(-1)^{e d}-i f_{d g}^{c} f_{e}^{a g}\right): j_{z}^{e} j_{\bar{z}}^{d}:(w) \\
& +g / 2\left(i f_{e g}^{c} f_{d}^{a g}(-1)^{e d}+i f_{d g}^{c} f^{a g}{ }_{e}\right): j_{z}^{e} j_{\bar{z}}^{d}:(w) \\
& \left.+i f^{c}{ }_{h x} f^{a x}{ }_{g}\left(c_{2}-g\right)\right) B^{g h}{ }_{e d}: j_{z}^{e} j_{\bar{z}}^{d}: \\
& +\mathcal{O}\left(f^{2}\right)
\end{aligned}
$$

where we have separated out (graded) symmetric and anti-symmetric terms. We now apply the super Jacobi identity to the first term in the third line and note that:

$$
\begin{aligned}
f_{g}^{c e} f_{d}^{a g} & =f_{g}^{c e} f_{d}^{g a}(-1)^{1+a+a d} \\
& =f^{e c}{ }_{g} f_{d}^{g a}{ }_{d}(-1)^{a+a d+e c} \\
& =-(-1)^{a+a d+e c+c d}\left((-1)^{a c} f_{g}^{e a} f_{d}^{g}{ }^{c}+(-1)^{a d} f_{d g}^{e} f^{g c a}\right),
\end{aligned}
$$

which leads to:

$$
\begin{aligned}
f_{e g}^{c} f_{d}^{a g}(-1)^{e d}-f_{d g}^{c} f_{e}^{a g}= & (-1)^{1+a+a d+e c+c d+e d+a c+e+g+g+1+c d} f_{d g}^{c} f_{e}^{a g} \\
& +(-1)^{1+a+a d+e c+c d+a d+g+e d+g+g+c a+e d} f_{g}^{a c} f_{d e}^{g} \\
& -f_{d g}^{c} f_{d e}^{a g} \\
= & -f^{a c}{ }_{g} f^{g}{ }_{d e} .
\end{aligned}
$$

Therefore, the third line cancels the first term in the first line and we are left with:

$$
\begin{aligned}
& \left(c_{-}(B)^{a c}{ }_{e d}+c_{+}(B)^{a c}{ }_{e d}\right): j_{z}^{e} j_{\bar{z}}^{d}:(w) \\
& \quad+g / 2\left(i f^{c}{ }_{e g} f^{a g}{ }_{d}(-1)^{e d}+i f^{c}{ }_{d g} f^{a g}{ }_{e}\right): j_{z}^{e} j_{\bar{z}}^{d}:(w) \\
& \quad-i f^{c}{ }_{h x} f^{a x}{ }_{g}\left(c_{2}-g\right) B^{g h}{ }_{e d}: j_{z}^{e} j_{\bar{z}}^{d}:+\mathcal{O}\left(f^{2}\right) .
\end{aligned}
$$

As expected the demand of the vanishing of this term gives the value of the tensor $B$ at the first non-trivial order in $f^{2}$ :

$$
B_{e d}^{a c}=-i \frac{g}{2\left(c_{+}+c_{-}\right)}\left(f_{e g}^{c} f_{d}^{a g}(-1)^{e d}+f_{d g}^{c} f_{e}^{a g}\right)+O\left(f^{4}\right) .
$$

5. A similar analysis for the other two first-order poles proportional respectively to $1 /(z-w)$ and $(z-w) /(\bar{z}-\bar{w})^{2}$ gives respectively the tensors $A$ and $C$ in equations $(2.5),(2.9)$. The details of the calculation are very similar to the calculation we just discussed.

Remarks on higher order terms in $f^{2}$. To discuss a few aspects of the higher order terms that we encountered, it is useful to define the following tensor:

$$
S_{e d}^{a c}=f_{e g}^{c} f_{d}^{a g}(-1)^{e d}+f_{d g}^{c} f_{e}^{a g} .
$$


It is manifestly graded symmetric in the lower indices. Let's also check that it is graded symmetric in the upper indices:

$$
\begin{aligned}
S_{e d}^{c a} & =f^{a}{ }_{e g} f_{d}^{c g}(-1)^{e d}+f_{d g}^{a} f_{e}^{c g} \\
& =f^{a g}{ }_{e}(-1)^{g+e g+1+e d} f_{d g}^{c}(-1)^{g d+1}+(-1)^{g+1+g d+1+e g} f_{e g}^{c} f^{a g}{ }_{d} \\
& =f^{a g}{ }_{e}(-1)^{a c} f_{d g}^{c}+(-1)^{a c} f^{c}{ }_{e g} f^{a g}{ }_{d} \\
& =(-1)^{a c} S^{a c}{ }_{e d} .
\end{aligned}
$$

Therefore, $S$ is a linear operator that acts on the space of (graded) symmetric two-tensors.

The higher order term in the last line in the above explicit calculation (D.16) gives rise to the square of the linear operator $S$. We computed it for $\operatorname{psl}(2 \mid 2)$ for which it simplifies to

$$
S_{g h}^{a c} S^{g h}{ }_{e d}=8\left(\kappa^{a c} \kappa_{d e}+\left(\delta_{e}^{a} \delta_{d}^{c}+(-1)^{e d} \delta_{d}^{a} \delta_{e}^{c}\right)\right) .
$$

We also have the equality $S^{3}=16 S$. When we take a supertrace of $S^{2}$, it can be shown to be zero because the superdimension of $\operatorname{psl}(2 \mid 2)$ is -2 .

Using some of these properties, it is clear that at higher order the structure of a pole in the $j^{a} \cdot M C^{c}$ OPE will look like:

$$
\ldots \kappa^{a c}: j_{e \bar{z}} j_{z}^{e}:+\ldots\left(: j_{\bar{z}}^{a} j_{z}^{c}:+(-1)^{a c}: j_{\bar{z}}^{c} j_{z}^{a}:\right)
$$

The first term is proportional to a component of the energy-momentum tensor (and to the kinetic term in the Lagrangian). The other term indicates that at higher order, we need a new four-tensor index structure in the current-current operator product expansion. At the same time, the special properties of the linear operator given above show that only few four-tensors will appear. It is certainly feasible to push the above calculation, and therefore the other calculations in the bulk of the paper to higher order.

\section{D.2 The Virasoro algebra from the current algebra}

In [4] it was shown that the Virasoro algebra emerges from the current algebra (2.5) via the Sugawara construction. More precisely it was argued that the normal ordered classical expression for the stress tensor:

$$
T=\frac{1}{2 c_{1}}: j_{L, z b} j_{L, z}^{b}:
$$

satisfies the OPEs:

$$
\begin{aligned}
T(z) j_{L, z}^{a}(w) & =\frac{j_{L, z}^{a}(w)}{(z-w)^{2}}+\frac{\partial j_{L, z}^{a}(w)}{z-w}+\mathcal{O}(z-w)^{0} \\
T(z) T(w) & =\frac{\operatorname{sdim}(G)}{2(z-w)^{4}}+\frac{T(w)}{(z-w)^{2}}+\frac{\partial T(w)}{z-w}+\mathcal{O}(z-w)^{0} .
\end{aligned}
$$

In this section, we fill a gap in the demonstration of equation (D.23). We reconsider the OPE between a current and the bilinear operator $: j_{L, z b} j_{L, z}^{b}:$. To perform this computation 
in [4] we truncated the current algebra at the order of the poles. We obtained:

$$
\begin{aligned}
j_{L, z}^{a}(z): j_{L, z b} j_{L, z}^{b}:(w)= & 2 c_{1} \frac{j_{L, z}^{a}(w)}{(z-w)^{2}}+c_{2} \frac{f_{b c}^{a}}{z-w}\left((-1)^{b c}: j_{L, z}^{b} j_{L, z}^{c}:+: j_{L, z}^{c} j_{L, z}^{b}:(w)\right) \\
& +\left(c_{2}-g\right) f_{b c}^{a} \frac{\bar{z}-\bar{w}}{(z-w)^{2}}\left((-1)^{b c}: j_{L, z}^{b} j_{L, \bar{z}}^{c}:+: j_{L, \bar{z}}^{c} j_{L, z}^{b}:(w)\right) \\
& +\ldots
\end{aligned}
$$

where the ellipses contain terms of order zero in the distance between the insertion points $z$ and $w$. We will now show that the subleading terms in the current algebra do not modify this result. Let us divide these terms into two sets. First we have the regular terms and the terms that multiply an $n^{\text {th }}$-derivative of a single current. These terms were already considered in [4] and it is straightforward to show that they do not modify (D.24). The second set contains the terms that multiply composites of (derivatives of) several currents (not including the regular terms). This includes for instance the current bilinears in equation (2.5). The crucial point is that all these terms come with a coefficient that contains at least two structure constants. This is a consequence of the discussion in appendix B. In full generality, a term in this second set may lead to the following type of contribution to (D.24):

$$
\begin{gathered}
\frac{T_{b{ }_{b} j_{L, z}^{b}}^{b}(w)}{(z-w)^{2}}+\frac{U^{a}{ }_{b} \partial j_{L, z}^{b}(w)}{z-w}+\frac{V_{b}^{a} \bar{\partial} j_{L, z}^{b}(w)(\bar{z}-\bar{w})}{(z-w)^{2}}+\frac{\bar{T}_{b}^{a} j_{L, \bar{z}}^{b}(w)(\bar{z}-\bar{w})}{(z-w)^{3}} \\
+\frac{\bar{U}_{b}^{a} \partial j_{L, \bar{z}}^{b}(w)(\bar{z}-\bar{w})}{(z-w)^{2}}+\frac{\bar{V}_{b}^{a} \bar{\partial} j_{L, \bar{z}}^{b}(w)(\bar{z}-\bar{w})^{2}}{(z-w)^{3}}+\frac{W^{a}{ }_{b c}: j_{L, z}^{c} j_{L, z}^{b}:(w)}{z-w} \\
+\frac{X_{b c}^{a}: j_{L, \bar{z}}^{c} j_{L, z}^{b}:(w)(\bar{z}-\bar{w})}{(z-w)^{2}}+\frac{Y_{b c}^{a}: j_{L, \bar{z}}^{c} j_{L, \bar{z}}^{b}:(w)(\bar{z}-\bar{w})^{2}}{(z-w)^{3}}
\end{gathered}
$$

where the tensors $T_{b}^{a}$, etc. are invariant two- and three-tensors made of contractions of structure constants. According to the argument of [2], any invariant two-tensor obtained by contracting at least one structure constant vanishes. Moreover any invariant threetensor obtained by contracting at least two structure constant also vanishes. Since all tensors appearing in (D.25) contain at least two structure constants that come from the current-current OPE, all these terms vanish. This completes the proof of equation (D.23).

\section{D.3 Currents as a primary fields of dimension one revisited}

The stress-energy tensor can be written either in terms of the left or of the right currents. As a consistency check on our formalism, we will compute in this appendix the OPE between the stress-energy tensor and the current components $j_{L, z}$ using the expression of the energy-momentum tensor $T$ in terms of the right currents:

$$
T(z)=\frac{1}{2 c_{3}}: j_{R, z}^{\bar{b}} j_{R, z}^{\bar{c}}:(w) \kappa_{\bar{c} \bar{b}}
$$

To proceed we use the OPEs between left and right currents (3.15), (3.16), as well as the OPEs between a current and the primary adjoint operator (3.10). Notice that the latter 
OPE may receive higher-order corrections in $f^{2}$. In the following we keep track only of the leading-order terms in $f^{2}$. The computation goes as follows:

$$
\begin{aligned}
j_{L, z}^{a}(z): j_{R, z}^{\bar{b}} j_{R, z}^{\bar{c}}:(w) \kappa_{\bar{c} \bar{b}}=\frac{c_{+} c_{-}}{c_{+}+c_{-}}\left(\frac{\left[: \phi^{a \bar{b}} j_{R, z}^{\bar{c}}:(w)+: j_{R, z}^{\bar{b}} \phi^{a \bar{c}}:(w)\right] \kappa_{\bar{c} \bar{b}}}{(z-w)^{2}}\right. \\
+\frac{c_{-}}{c_{+}+c_{-}} \frac{\left[: \partial \phi^{a \bar{b}} j_{R, z}^{\bar{c}}:(w)+: j_{R, z}^{\bar{b}} \partial \phi^{a \bar{c}}:(w)\right] \kappa_{\bar{c} \bar{b}}}{z-w} \\
\left.+\frac{c_{-}}{c_{+}+c_{-}} \frac{\left[: \bar{\partial} \phi^{a \bar{b}} j_{R, z}^{\bar{c}}:(w)+: j_{R, z}^{\bar{b}} \bar{\partial} \phi^{a \bar{c}}:(w)\right] \kappa_{\bar{c} \bar{b}}(\bar{z}-\bar{w})}{(z-w)^{2}}\right)
\end{aligned}
$$

where a triple pole vanishes since it is proportional to the contraction of a structure constant with the metric. We have to treat carefully the normal-ordered operators appearing in the previous expression. The central point is the property:

$$
: j_{R}^{\bar{a}} \phi^{b \bar{b}}:-: \phi^{b \bar{b}} j_{R}^{\bar{a}}: \propto f^{\bar{a} \bar{b}}
$$

and similarly for the left currents. This property follows from the OPE between the current and the adjoint primary (3.10). Thus we can deal with the first line easily, and using equation (3.6) we obtain:

$$
\frac{\left[: \phi^{a \bar{b}} j_{R, z}^{\bar{c}}:(w)+: j_{R, z}^{\bar{b}} \phi^{a \bar{c}}:(w)\right] \kappa_{\bar{b} \bar{c}}}{(z-w)^{2}}=-\frac{c_{-}}{c_{+}} \frac{2 j_{L, z}^{a}(w)}{(z-w)^{2}} .
$$

Now let us consider the second line. We use equation (3.11):

$$
\partial \phi^{a \bar{a}}=-\frac{i f^{\bar{a}} \bar{b} \bar{c}}{c_{-}} j_{R, z}^{\bar{c}} \phi^{a \bar{b}}
$$

Notice that we do not need the normal ordering symbol on the right-hand side since (at leading order in $f^{2}$ ) there is no singular term to discard in the OPEs (3.10). Thus we rewrite the second line as:

$$
\begin{aligned}
& \frac{c_{-}}{c_{+}+c_{-}} \frac{\left[: \partial \phi^{a \bar{b}} j_{R, z}^{\bar{c}}:(w)+: j_{R, z}^{\bar{b}} \partial \phi^{a \bar{c}}:(w)\right] \kappa_{\bar{b} \bar{c}}}{z-w} \\
& \quad=\frac{-i}{c_{+}+c_{-}} \frac{\left[: f^{\bar{b}} \overline{d \bar{e}} j_{R, z}^{\bar{e}} \phi^{a \bar{d}} j_{R, z}^{\bar{c}}:(w)+: j_{R, z}^{\bar{b}} f^{\bar{c}} \overline{d \bar{e}} j_{R, z}^{\bar{e}} \phi^{a \bar{d}}:(w)\right] \kappa_{\bar{b} \bar{c}}}{z-w} \\
& \quad=\frac{-i f_{\bar{c} \bar{d} \bar{e}}}{c_{+}+c_{-}} \frac{: j_{R, z}^{\bar{e}} j_{R, z}^{\bar{c}} \phi^{a \bar{d}}:(w)+: j_{R, z}^{\bar{c}} j_{R, z}^{\bar{e}} \phi^{a \bar{d}}:(w)}{z-w}
\end{aligned}
$$

where we used the property (D.28) again in the last step to commute the adjoint operator and the current in the normal ordered triple operator. Now thanks to the anti-symmetry of the structure constants this term vanishes. We can perform similar manipulations on the third line:

$$
\frac{c_{-}}{c_{+}+c_{-}} \frac{\left[: \bar{\partial} \phi^{a \bar{b}} j_{R, z}^{\bar{c}}:(w)+: j_{R, z}^{\bar{b}} \bar{\partial} \phi^{a \bar{c}}:(w)\right] \kappa_{\bar{b} \bar{c}}(\bar{z}-\bar{w})}{(z-w)^{2}} .
$$


The first operator can be rewritten as:

$$
: \bar{\partial} \phi^{a \bar{b}} j_{R, z}^{\bar{c}}:(w) \kappa_{\bar{b} \bar{c}}=-\frac{i f_{\bar{c} \bar{d} \bar{e}}}{c_{+}}: j_{R, \bar{z}}^{\bar{e}} j_{R, z}^{\bar{c}} \phi^{a \bar{d}}:(w)
$$

and the second one as:

$$
: j_{R, z}^{\bar{b}} \bar{\partial} \phi^{a \bar{c}}:(w) \kappa_{\bar{b} \bar{c}}=-\frac{i f_{\bar{c} \bar{d} \bar{e}}}{c_{+}}: j_{R, z}^{\bar{c}} j_{R, \bar{z}}^{\bar{e}} \phi^{a \bar{d}}:(w)=: \bar{\partial} \phi^{a \bar{b}} j_{R, z}^{\bar{c}}:(w) \kappa_{\bar{b} \bar{c}}
$$

where in the last step we used that $f_{\bar{c} \bar{d} \bar{e}}: j_{R, z}^{\bar{c}} j_{R, \bar{z}}^{\bar{e}}:=f_{\bar{c} \bar{d} \bar{e}}: j_{R, \bar{z}}^{\bar{e}} j_{R, z}^{\bar{c}}:$. Therefore the two operators present on the third line are the same. Now we can use the Maurer-Cartan equation and current conservation for the right currents to rewrite them as:

$$
-\frac{i f_{\bar{c} \bar{d} \bar{e}}}{c_{+}}: j_{R, \bar{z}}^{\bar{e}} j_{R, z}^{\bar{c}} \phi^{a \bar{d}}:(w)=-\frac{c_{+}+c_{-}}{c_{+}}: \bar{\partial} j_{R, z}^{\bar{b}} \phi^{a \bar{d}}:(w) \kappa_{\bar{b} \bar{d}}
$$

Therefore, we can rewrite the third line as:

$$
\frac{c_{-}}{c_{+}+c_{-}} \frac{2\left(c_{+}+c_{-}\right)}{c_{-}} \frac{\left[: \bar{\partial} j_{R, z}^{\bar{b}} \phi^{a \bar{c}}:(w)+: j_{R, z}^{\bar{b}} \bar{\partial} \phi^{a \bar{c}}:(w)\right] \kappa_{\bar{b} \bar{c}}(\bar{z}-\bar{w})}{(z-w)^{2}}=-2 \frac{c_{+}}{c_{-}} \frac{\bar{\partial} j_{L, z}^{a}(w)(\bar{z}-\bar{w})}{(z-w)^{2}}
$$

where we used equation (3.6) once more. Gathering all terms, we obtain:

$$
j_{L, z}^{a}(z): j_{R, z}^{\bar{b}} j_{R, z}^{\bar{c}}:(w) \kappa_{\bar{b} \bar{c}}=-\frac{2 c_{-}^{2}}{c_{+}+c_{-}}\left(\frac{j_{L, z}^{a}(w)}{(z-w)^{2}}+\frac{\bar{\partial} j_{L, z}^{a}(w)(\bar{z}-\bar{w})}{(z-w)^{2}}\right)
$$

which we can finally rewrite in the expected form:

$$
T(w) j_{L, z}^{a}(z)=\frac{1}{2 c_{3}}: j_{R, z}^{\bar{b}} j_{R, z}^{\bar{c}}:(w) \kappa_{\bar{c} \bar{c}} j_{L, z}^{a}(z)=\frac{j_{L, z}^{a}(w)}{(w-z)^{2}}+\frac{\partial j_{L, z}^{a}(w)}{w-z},
$$

thus completing our consistency check.

\section{D.4 The associativity of the current algebra}

In this appendix we address the issue of the associativity of the current algebra (2.5). We will prove the associativity of this current algebra at the first non-trivial order in $f^{2}$.

The OPE $j_{L, z}^{a}(z) j_{L, z}^{b}(w) j_{L, z}^{c}(x)$. First we consider the OPE between three $z$ components of the left-current:

$$
j_{L, z}^{a}(z) j_{L, z}^{b}(w) j_{L, z}^{c}(x) .
$$

We will compute this OPE using the current algebra (2.5) at the order of the poles. Moreover we will only compute the lowest-order terms in the $f^{2}$ expansion. In this case these are terms of order $f^{-2}$. To prove associativity we will first compute the OPE between the first two currents, then compute the OPE of the result with the third current, and show that the result is invariant under permutation of the currents. We start out with:

$$
\begin{gathered}
j_{L, z}^{a}(z) j_{L, z}^{b}(w) j_{L, z}^{c}(x)=\left(\frac{c_{1} \kappa^{a b}}{(z-w)^{2}}+\frac{{ }_{c} f_{d}^{a b} j_{L, z}^{d}(w)}{z-w}+\frac{\left(c_{2}-g\right) f_{d}^{a b} j_{L, \bar{z}}^{d}(w)(\bar{z}-\bar{w})}{(z-w)^{2}}\right. \\
\left.+: j_{L, z}^{a}(z) j_{L, z}^{b}(w):+\ldots\right) j_{L, z}^{c}(x)
\end{gathered}
$$


The ellipses stand for lower-order terms in the OPEs, that we do not keep track of. We obtain:

$$
j_{L, z}^{a}(z) j_{L, z}^{b}(w) j_{L, z}^{c}(x)=\frac{c_{1} \kappa^{a b} j_{L, z}^{c}(x)}{(z-w)^{2}}+\frac{c_{1} c_{2} f^{a b c}}{(z-w)(w-x)^{2}}+: j_{L, z}^{a}(z) j_{L, z}^{b}(w): j_{L, z}^{c}(x)+\ldots
$$

up to a contact terms. We now have to compute the OPE involving the regular operator $: j_{L, z}^{a}(z) j_{L, z}^{b}(w):$. In order to use the techniques presented in appendix A we rewrite both currents as being evaluated at the point $w$ :

$$
: j_{L, z}^{a}(z) j_{L, z}^{b}(w):=\sum_{n, \bar{n}=0}^{\infty} \frac{(z-w)^{n}}{n !} \frac{(\bar{z}-\bar{w})^{\bar{n}}}{\bar{n} !}:\left(\partial^{n} \bar{\partial}^{\bar{n}} j_{L, z}^{a}\right) j_{L, z}^{b}:(w)
$$

Let us now consider the OPE of one of these composite operators with the current $j_{L, z}^{c}(x)$ :

$$
\begin{aligned}
j_{L, z}^{c}(x) & :\left(\partial^{n} \bar{\partial}^{\bar{n}} j_{L, z}^{a}\right) j_{L, z}^{b}:(w)=j_{L, z}^{c}(x) \lim _{: y \rightarrow w:} \partial_{y}^{n} \bar{\partial}_{y}^{\bar{n}} j_{L, z}^{a}(y) j_{L, z}^{b}(w) \\
= & \lim _{: y \rightarrow w:} \partial_{y}^{n} \bar{\partial}_{y}^{\bar{n}}\left[\left(\frac{c_{1} \kappa^{c a}}{(x-y)^{2}}+\frac{c_{2} f^{c a}{ }_{d} j_{L, z}^{d}(y)}{x-y}+\frac{\left(c_{2}-g\right) f^{c a}{ }_{d} j_{L, \bar{z}}^{d}(y)(\bar{x}-\bar{y})}{(x-y)^{2}}\right.\right. \\
& \left.+\sum_{m, \bar{m}=0}^{\infty} \frac{(x-y)^{m}}{m !} \frac{(\bar{x}-\bar{y})^{\bar{m}}}{\bar{m} !}:\left(\partial^{m} \bar{\partial}^{\bar{m}} j_{L, z}^{c}\right) j_{L, z}^{a}:(y)+\mathcal{O}\left(f^{2}\right)\right) j_{L, z}^{b}(w) \\
& \left.+j_{L, z}^{a}(y)\left(\frac{c_{1} \kappa^{c b}}{(x-w)^{2}}+\ldots\right)\right] \\
= & \lim _{: y \rightarrow w:} \partial_{y}^{n} \bar{\partial}_{y}^{\bar{n}}\left[\frac{c_{1} \kappa^{c a} j_{L, z}^{b}(w)}{(x-y)^{2}}+\frac{c_{1} c_{2} f^{c a b}}{(x-y)(y-w)^{2}}+\frac{c_{1} \kappa^{c b} j_{L, z}^{a}(y)}{(x-w)^{2}}+. .\right]
\end{aligned}
$$

where the ellipses in the last line contains singular terms that comes from the OPE between the regular operators and the current in the third line of the previous computation. These terms in this OPE will be removed by the regular limit $: y \rightarrow w:$. In order to compute the action of the derivatives more conveniently, we rewrite the second term in the last line as:

$$
\frac{c_{1} c_{2} f^{c a b}}{(x-y)(y-w)^{2}}=c_{1} c_{2} f^{c a b} \sum_{p=0}^{\infty} \frac{(y-w)^{p-2}}{(x-w)^{p+1}}
$$

Thus we obtain:

$$
\begin{aligned}
j_{L, z}^{c}(x):\left(\partial^{n} \bar{\partial}^{\bar{n}} j_{L, z}^{a}\right) j_{L, z}^{b}:(w)=\lim _{y \rightarrow w:}\left[\delta_{\bar{n}, 0} \frac{(n+1) !}{(x-y)^{n+2}} c_{1} \kappa^{c a} j_{L, z}^{b}(w)\right. \\
\left.\quad+\delta_{\bar{n}, 0} \sum_{p=0}^{\infty} \frac{(p-2) \ldots(p-2-n+1)(y-w)^{p-2-n}}{(x-w)^{p+1}} c_{1} c_{2} f^{c a b}+\frac{c_{1} \kappa^{c b} \partial^{n} \bar{\partial}^{\bar{n}} j_{L, z}^{a}(y)}{(x-w)^{2}}+\ldots\right] \\
=\delta_{\bar{n}, 0} \frac{(n+1) !}{(x-w)^{n+2}} c_{1} \kappa^{c a} j_{L, z}^{b}(w)+\delta_{\bar{n}, 0} \frac{n !}{(x-w)^{n+3}} c_{1} c_{2} f^{c a b}+\frac{c_{1} \kappa^{c b} \partial^{n} \bar{\partial}^{\bar{n}} j_{L, z}^{a}(w)}{(x-w)^{2}}+\ldots
\end{aligned}
$$


Resumming the series, we get:

$$
\begin{aligned}
: j_{L, z}^{a}(z) j_{L, z}^{b}(w): j_{L, z}^{c}(x)= & \sum_{n, \bar{n}=0}^{\infty} \frac{(z-w)^{n}}{n !} \frac{(\bar{z}-\bar{w})^{\bar{n}}}{\bar{n} !}\left[\delta_{\bar{n}, 0} \frac{(n+1) !}{(x-w)^{n+2}} c_{1} \kappa^{c a} j_{L, z}^{b}(w)\right. \\
& \left.+\delta_{\bar{n}, 0} \frac{n !}{(x-w)^{n+3}} c_{1} c_{2} f^{c a b}+\frac{c_{1} \kappa^{c b} \partial^{n} \bar{\partial}^{\bar{n}} j_{L, z}^{a}(w)}{(x-w)^{2}}+\ldots\right] \\
= & \frac{c_{1} \kappa^{c a} j_{L, z}^{b}(w)}{(x-z)^{2}}+\frac{c_{1} c_{2} f^{c a b}}{(x-z)(x-w)^{2}}+\frac{c_{1} \kappa^{c b} j_{L, z}^{a}(z)}{(x-w)^{2}}+\ldots
\end{aligned}
$$

After gathering all terms, we obtain:

$$
\begin{aligned}
j_{L, z}^{a}(z) j_{L, z}^{b}(w) j_{L, z}^{c}(x)= & \frac{c_{1} \kappa^{a b} j_{L, z}^{c}(x)}{(z-w)^{2}}+\frac{c_{1} c_{2} f^{a b c}}{(z-w)(w-x)^{2}}+\frac{c_{1} \kappa^{c a} j_{L, z}^{b}(w)}{(x-z)^{2}} \\
& +\frac{c_{1} c_{2} f^{c a b}}{(x-z)(x-w)^{2}}+\frac{c_{1} \kappa^{c b} j_{L, z}^{a}(z)}{(x-w)^{2}}+\ldots \\
= & \frac{c_{1} c_{2} f^{a b c}}{(z-x)(x-w)(w-z)}+\frac{c_{1} \kappa^{a b} j_{L, z}^{c}(x)}{(z-w)^{2}} \\
& +\frac{c_{1} \kappa^{c a} j_{L, z}^{b}(w)}{(x-z)^{2}}+\frac{c_{1} \kappa^{c b} j_{L, z}^{a}(z)}{(x-w)^{2}}+\mathcal{O}\left(f^{0}\right)+\ldots
\end{aligned}
$$

which is manifestly invariant under permutation of the currents.

The OPE $j_{L, z}^{a}(z) j_{L, z}^{b}(w) j_{L, \bar{z}}^{c}(x)$. We now consider the OPE involving two $z$ components and one $\bar{z}$-component of the left current:

$$
j_{L, z}^{a}(z) j_{L, z}^{b}(w) j_{L, \bar{z}}^{c}(x) .
$$

First we will take first the OPE between the two $z$-components of the current:

$$
\begin{aligned}
& {\left[j_{L, z}^{a}(z) j_{L, z}^{b}(w)\right] j_{L, \bar{z}}^{c}(x)} \\
& \quad=\left(\frac{c_{1} \kappa^{a b}}{(z-w)^{2}}+\frac{c_{2} f^{a b}{ }_{d} j_{L, z}^{d}(w)}{z-w}+\frac{\left(c_{2}-g\right) f^{a b}{ }_{d j_{L, \bar{z}}}^{d}(w)(\bar{z}-\bar{w})}{(z-w)^{2}}+: j_{L, z}^{a}(z) j_{L, z}^{b}(w):+\ldots\right) j_{L, \bar{z}}^{c}(x) \\
& \quad=\frac{c_{1} \kappa^{a b} j_{L, \bar{z}}^{c}(x)}{(z-w)^{2}}+\frac{c_{3}\left(c_{2}-g\right) f^{a b c}(\bar{z}-\bar{w})}{(z-w)^{2}(\bar{w}-\bar{x})^{2}}+: j_{L, z}^{a}(z) j_{L, z}^{b}(w): j_{L, \bar{z}}^{c}(x)+\ldots
\end{aligned}
$$

The OPE involving the composite operator does not produces any term of order $f^{-2}$, thus we obtain:

$$
\left[j_{L, z}^{a}(z) j_{L, z}^{b}(w)\right] j_{L, \bar{z}}^{c}(x)=\frac{c_{1} \kappa^{a b} j_{L, \bar{z}}^{c}(x)}{(z-w)^{2}}+\frac{c_{3}\left(c_{2}-g\right) f^{a b c}(\bar{z}-\bar{w})}{(z-w)^{2}(\bar{w}-\bar{x})^{2}}+\mathcal{O}\left(f^{0}\right)+\ldots
$$


Now let us perform the same computation taking first the OPE between one $z$ component and one $\bar{z}$-component of the current:

$$
\begin{aligned}
j_{L, z}^{a}(z) & {\left[j_{L, z}^{b}(w) j_{L, \bar{z}}^{c}(x)\right] } \\
& =j_{L, z}^{a}(z)\left(\frac{\left(c_{4}-g\right) f^{b c}{ }_{d} j_{L, z}^{d}(x)}{\bar{w}-\bar{x}}+\frac{\left(c_{2}-g\right) f^{b c}{ }_{d} j_{L, \bar{z}}^{d}(x)}{(w-x)}+: j_{L, z}^{b}(w) j_{L, \bar{z}}^{c}(x):+\ldots\right) \\
& =\frac{c_{1}\left(c_{4}-g\right) f^{a b c}}{(z-w)^{2}(\bar{w}-\bar{x})}+j_{L, z}^{a}(z): j_{L, z}^{b}(w) j_{L, \bar{z}}^{c}(x):+\ldots \\
& =\frac{c_{1}\left(c_{4}-g\right) f^{a b c}}{(z-w)^{2}(\bar{w}-\bar{x})}+\frac{c_{1} \kappa^{a b} j_{L, \bar{z}}^{c}(x)}{(z-w)^{2}}+\ldots
\end{aligned}
$$

Thanks to the relations between the coefficients of the current algebra:

$$
c_{1}\left(c_{4}-g\right)=c_{3}\left(c_{2}-g\right)
$$

we find that the current algebra is indeed associative at the order at which we performed the computation. The coordinate dependence does not match exactly since we did not take into account the terms containing derivatives of the currents that appear in the current algebra as subleading terms. It is interesting to pursue the full proof of associativity.

\section{D.5 The holomorphy of the stress-tensor}

In this appendix we address the issue of the holomorphy of the stress-tensor: ${ }^{6}$

$$
T(z)=\frac{1}{2 c_{1}} \kappa_{a b}: j_{L, z}^{b} j_{L, z}^{a}:(z) .
$$

Since the $z$-component of the left-current is not holomorphic away from the WZW point, it is not obvious that the stress-tensor will be holomorphic in the quantum theory. The anti-holomorphic derivative of the stress-tensor reads:

$$
\bar{\partial} T(z)=\frac{1}{2 c_{1}} \kappa_{a b}\left(: \bar{\partial} j_{L, z}^{b} j_{L, z}^{a}:(z)+: j_{L, z}^{b} \bar{\partial} j_{L, z}^{a}:(z)\right) .
$$

To continue the computation we combine current conservation with the Maurer-Cartan equation to write the anti-holomorphic derivative of the $z$-component of the current in terms of a bilinear:

$$
\bar{\partial} j_{L, z}^{a}=-i f^{2} f_{b c}^{a}: j_{L, z}^{c} j_{L, \bar{z}}^{b}: .
$$

Since all the poles in the OPE between $j_{L, z}^{c}$ and $j_{L, \bar{z}}^{b}$ vanish when contracted with the structure constant $f_{b c}^{a}$, we can also write:

$$
\bar{\partial} j_{L, z}^{a}=-i f^{2} f_{b c}^{a}: j_{L, \bar{z}}^{b} j_{L, z}^{c}: .
$$

Thus using successively the last two equations we obtain:

$$
\bar{\partial} T(z)=\frac{-i f^{2}}{2 c_{1}} f_{a b c}\left(:: j_{L, z}^{c} j_{L, \bar{z}}^{b}: j_{L, z}^{a}:(z)+: j_{L, z}^{a}: j_{L, \bar{z}}^{b} j_{L, z}^{c}::(z)\right) .
$$

\footnotetext{
${ }^{6}$ We would like to thank Matthias Gaberdiel for raising the issue.
} 
Now let us consider the composite operator $:: j_{L, z}^{c} j_{L, \bar{z}}^{b}: j_{L, z}^{a}:(z)$. It is defined as the regular term in the OPE between $: j_{L, z}^{c} j_{L, \bar{z}}^{b}$ : and $j_{L, z}^{a}$. We will show that we have:

$$
f_{a b c}:: j_{L, z}^{c} j_{L, \bar{z}}^{b}: j_{L, z}^{a}:(z)=f_{a b c}: j_{L, z}^{c} j_{L, \bar{z}}^{b} j_{L, z}^{a}:(z)
$$

where the operator : $j_{L, z}^{c} j_{L, z}^{b} j_{L, z}^{a}$ : is defined as the regular term in the OPE of the three currents $j_{L, z}^{c}, j_{L, \bar{z}}^{b}$ and $j_{L, z}^{a}$. The difference between the operators on the left-hand side and the right-hand side of equation (D.57) comes from the non-regular terms in the OPE between $j_{L, z}^{c}$ and $j_{L, \bar{z}}^{b}$. The crucial point is that all these terms vanish when contracted with the structure constant $f_{a b c}$ :

$$
f_{a b c}\left[j_{L, z}^{c}(z) j_{L, \bar{z}}^{b}(w)-: j_{L, z}^{c}(z) j_{L, \bar{z}}^{b}(w):\right]=0 .
$$

This can be checked via the current algebra OPEs (2.5) order by order in $f^{2}$. In equation (2.5) the current algebra is given up to terms of order $f^{4}$, and thus one can prove the previous statement up to terms of order $f^{4}$. Indeed, all tensors that appear in the current algebra (2.5) vanish upon double contraction with a structure constant:

$$
f_{a b c}\left[\kappa^{c b}, f^{c b d}, A_{d e}^{c b}, B_{d e}^{c b}, C_{d e}^{c b}\right]=0
$$

The non-degenerate metric $\kappa^{c b}$ and the tensors $A_{d e}^{c b}, B_{d e}^{c b}, C_{d e}^{c b}$ are graded-symmetric in the indices $c, b$. Moreover the double contraction of the structure constant vanishes since the dual Coxeter number of the Lie super algebra vanishes. This concludes the proof of equation (D.57) up to terms of order $f^{4}$. Let us mention that the same equation (D.57) also guaranties the quantum integrability of the model up to this order, as discussed in section 7 . The same argument leads to the equality:

$$
f_{a b c}: j_{L, z}^{a}: j_{L, \bar{z}}^{b} j_{L, z}^{c}::(z)=f_{a b c}: j_{L, z}^{a} j_{L, \bar{z}}^{b} j_{L, z}^{c}:(z) .
$$

Thus we have:

$$
\bar{\partial} T(z)=\frac{-i f^{2}}{2 c_{1}} f_{a b c}\left(: j_{L, z}^{c} j_{L, \bar{z}}^{b} j_{L, z}^{a}:(z)+: j_{L, z}^{a} j_{L, \bar{z}}^{b} j_{L, z}^{c}:(z)\right)=0
$$

which vanishes thanks to the (graded) anti-symmetry of the structure constants. It would be interesting to have a non-perturbative understanding of the consistency of the normalordering and the holomorphy of the energy-momentum tensor.

\section{E Details on primary operators}

\section{E.1 Behavior of current primaries under perturbation of the kinetic term}

In this section we will show that current primaries at a given point of moduli space remain current primaries after perturbation of the kinetic term. More precisely we will show that if an operator $\phi$ satisfies the OPEs (4.1) at a given point of moduli space, then it also satisfies the same OPEs after exactly marginal deformation of the theory. This implies that it is consistent to think of a current primary as being the group element taken in a given 
representation, at any point of the moduli space. It also proves the claim in section 4 that the affine primary fields at the WZW points become current primaries after deformation of the theory.

For convenience let us recall the OPEs that define a primary operator $\phi$ :

$$
\begin{aligned}
& j_{L, z}^{a}(z) \phi(w)=-\frac{c_{+}}{c_{+}+c_{-}} t^{a} \frac{\phi(w)}{z-w}+\text { less singular } \\
& j_{L, \bar{z}}^{a}(z) \phi(w)=-\frac{c_{-}}{c_{+}+c_{-}} t^{a} \frac{\phi(w)}{\bar{z}-\bar{w}}+\text { less singular. }
\end{aligned}
$$

We assume that these OPEs hold at a given point of moduli space $\left(f^{2}, k\right)$. Then we perturb the kinetic term: $f^{2} \rightarrow f^{2}+\epsilon$ and we compute the way the OPEs (E.1) are modified. A procedure to compute OPEs in conformal perturbation theory was given in [4]. Here we will only compute the deformation of the OPEs (E.1) up to first order in $\epsilon$. The prescription is to compute first the OPE between the current and the perturbation of the action, and then to compute the OPE of the result with the field $\phi$. We begin with the first step of this procedure, for the first OPE in (E.1). The OPE between the current and the marginal operator can be computed thanks to the current algebra (2.5):

$$
\begin{aligned}
j_{L, z}^{a}(z) & \frac{\epsilon}{4 \pi f^{4}} \int d^{2} x \kappa_{c b}: \frac{j_{L, z}^{b}}{c_{+}} \frac{j_{L, \bar{z}}^{c}}{c_{-}}:(x) \\
& =\frac{\epsilon}{4 \pi f^{4} c_{+} c_{-}} \int d^{2} x\left(c_{1} \frac{j_{L, \bar{z}}^{a}(x)}{(z-x)^{2}}+\tilde{c} j_{L, z}^{a}(x) 2 \pi \delta^{(2)}(z-x)+\ldots\right) .
\end{aligned}
$$

The ellipses contains higher-order terms both in $f^{2}$ and in the distance between $z$ and $x$. We will not keep track of these terms for the time being, and we will comment on their relevance at the end of the computation. We now have to take the OPE of the previous result with the primary field $\phi$. We obtain:

$$
\begin{aligned}
\frac{\epsilon}{4 \pi f^{4} c_{+} c_{-}} & \int d^{2} x\left(-\frac{c_{1} c_{-}}{c_{+}+c_{-}} t^{a} \frac{\phi(w)}{(z-x)^{2}(\bar{x}-\bar{w})}-\frac{\tilde{c} c_{+}}{c_{+}+c_{-}} t^{a} \frac{\phi(w)}{x-w} \delta^{(2)}(z-x)+\ldots\right) \\
& =\frac{\epsilon}{2 f^{4} c_{+} c_{-}}\left(\frac{c_{1} c_{-}}{c_{+}+c_{-}}-\frac{\tilde{c} c_{+}}{c_{+}+c_{-}}\right) t^{a} \frac{\phi(w)}{z-w}+\ldots \\
& =-\epsilon c_{+} t^{a} \frac{\phi(w)}{z-w}+\text { less singular, }
\end{aligned}
$$

where we used the explicit value of the coefficients (2.6). As claimed, the structure of the OPEs (E.1) is unaltered after perturbation of the kinetic term. It is also straightforward to check that (taking into account the renormalization of the currents) the perturbation $f^{2} \rightarrow f^{2}+\epsilon$ induces a deformation of the coefficients in (E.1) that matches the result obtained at first order in $\epsilon$.

Now let us come back to the terms we discarded in equation (E.2). They contain the contribution to this computation from the poles and less singular terms in the current algebra (2.5). All these terms are (composites of) currents. It follows from (E.1) and from dimensional analysis that in the OPE between any one of these terms and the primary field $\phi$, the most singular term that may arise multiplies the operator $\phi$. Here we assume that all 
terms appearing in the OPE (E.1) can be written as composites of currents with the field $\phi$. Thus if any of these terms has any effect on the previous computation, it may at worse modify the coefficient obtained in (E.3). On the other hand, as was mentioned in section 4, the coefficients in (E.1) are fixed by demanding compatibility with current conservation and the Maurer-Cartan equation. Since these coefficients were already recovered in (E.3) it follows that the term we discarded in equation (E.2) indeed has no effect on the result of the computation. This can also be checked by hand for the terms that are explicitly given in equation (2.5).

\section{E.2 Current-primary OPE at order $f^{2}$}

Equation (4.1) gives the OPE between a current and a primary field at leading order. According to the discussion of section 5 it is possible to compute the higher-order terms thanks to current conservation and the Maurer-Cartan equation. In this appendix we perform the computation of the first correction to the OPE (4.1), which leads to the OPE (4.2) in the bulk of the paper.

The terms on the right-hand side of the OPE (4.1) are of order $f^{0}$. We will now compute the current-primary OPE at order $f^{2}$. Following the discussion of appendix B we make the following educated ansatz for the OPEs between the left-currents and a primary field $\phi$ :

$$
\begin{aligned}
j_{L, z}^{a}(z) \phi(w)= & -\frac{c_{+}}{c_{+}+c_{-}} \frac{t^{a} \phi(w)}{z-w}+: j_{L, z}^{a} \phi:(w) \\
& +A^{a}{ }_{c} \log |z-w|^{2}: j_{L, z}^{c} \phi:(w)+B^{a}{ }_{c} \frac{\bar{z}-\bar{w}}{z-w}: j_{L, \bar{z}}^{c} \phi:(w)+\mathcal{O}\left(f^{4}\right) \\
j_{L, \bar{z}}^{a}(z) \phi(w)= & -\frac{c_{-}}{c_{+}+c_{-}} \frac{t^{a} \phi(w)}{\bar{z}-\bar{w}}+: j_{L, \bar{z}}^{a} \phi:(w) \\
& +D^{a}{ }_{c} \log |z-w|^{2}: j_{L, \bar{z}}^{c} \phi:(w)+C^{a}{ }^{c} \frac{z-w}{\bar{z}-\bar{w}}: j_{L, z}^{c} \phi:(w)+\mathcal{O}\left(f^{4}\right) .
\end{aligned}
$$

We expect the coefficients $A^{a}{ }_{c}, C^{a}{ }_{c}, B^{a}{ }_{c}, D^{a}{ }_{c}$ to be of order $f^{2}$. We will check that the coefficient of the first-order poles are not modified. As explained in section 5 the demand of consistency with current conservation (5.4) imposes that the terms in the $j_{L, \bar{z}}^{a}(z) \phi(w)$ OPE can be deduced from the terms in the $j_{L, z}^{a}(z) \phi(w)$ :

$$
A^{a}{ }_{c}+C^{a}{ }_{c}=0=B^{a}{ }_{c}+D^{a}{ }_{c} .
$$

To get further constraints on the tensors $A^{a}{ }_{c}$ and $B^{a}{ }_{c}$ we ask for the vanishing of the first-order poles in the OPE between the operator $\phi$ and the Maurer-Cartan operator, that we write as in (C.10):

$$
\left[\bar{\partial} j_{L, z}^{a}(z)+i f^{2} f_{b c}^{a}: j_{L, z}^{c} j_{L, \bar{z}}^{b}:(z)\right] \phi(w)=0 .
$$

The first part of this OPE is:

$$
\bar{\partial} j_{L, z}^{a}(z) \phi(w)=A^{a}{ }_{c} \frac{: j_{L, z}^{c} \phi:(w)}{\bar{z}-\bar{w}}+B^{a}{ }_{c} \frac{: j_{L, \bar{z}}^{c} \phi:(w)}{z-w}+\mathcal{O}\left(f^{4}\right) .
$$


The simple poles in the previous expression should be canceled by the simple poles in the OPE between the composite operator $i f^{2} f_{b c}^{a}: j_{L, z}^{c} j_{L, \bar{z}}^{b}$ : and the operator $\phi$. Notice that because of the factors $f^{2}$ multiplying the composite operator, we only need to compute the OPE at order $f^{0}$. We calculate:

$$
\begin{aligned}
\phi(w)\left[i f^{2} f_{b c}^{a}: j_{L, z}^{c} j_{L, \bar{z}}^{b}:(z)\right]= & i f^{2} f_{b c}^{a} \lim _{: x \rightarrow z:} \phi(w) j_{L, z}^{c}(x) j_{L, \bar{z}}^{b}(z) \\
= & i f^{2} f_{b c}^{a} \lim _{: x \rightarrow z:}\left\{\left[-\frac{c_{+}}{c_{+}+c_{-}} \frac{t^{c} \phi(w)}{x-w}+: j_{L, z}^{c} \phi:(w) \ldots\right] j_{L, \bar{z}}^{b}(z)\right. \\
& \left.+j_{L, z}^{c}(x)\left[-\frac{c_{-}}{c_{+}+c_{-}} \frac{t^{b} \phi(w)}{\bar{z}-\bar{w}}+: j_{L, \bar{z}}^{b} \phi:(w)+\ldots\right]\right\} \quad \text { (E.8) }
\end{aligned}
$$

To proceed according to the prescription of appendix A we have to expand the fields in the first line (respectively the second line) in the neighborhood of the point $x$ (respectively $z$ ). Then we have to perform the remaining OPEs between the currents and the (derivatives of) the primary field $\phi$. Notice however that all the terms proportional to $f^{a}{ }_{c b} t^{b} t^{c}=$ $\frac{i}{2} f_{c b}^{a} f^{b c}{ }_{d} t^{d}$ do vanish. Only the regular term in the current-primary OPE will contribute to the result at order $f^{0}$. Moreover it is straightforward to check that the terms proportional to $A^{a}{ }_{c}$ and $B^{a}{ }_{c}$ in the previous OPE do not contribute at order $f^{0}$. We obtain:

$$
\phi(w)\left[i f^{2} f_{b c}^{a}: j_{L, z}^{c} j_{L, \bar{z}}^{b}:(z)\right]=-i f^{2} f_{b c}^{a}\left(\frac{c_{+}}{c_{+}+c_{-}} \frac{t^{c}: j_{L, \bar{z}}^{b} \phi:(z)}{z-w}+\frac{c_{-}}{c_{+}+c_{-}} \frac{t^{b}: j_{L, z}^{c} \phi:(z)}{\bar{z}-\bar{w}}+\ldots\right) .
$$

where the ellipses contains terms of order $f^{4}$ as well as terms of order zero in the distance between $z$ and $w$. Gathering terms, we conclude that we have the equalities:

$$
\begin{aligned}
A^{a}{ }_{c} & =\frac{c_{-}}{\left(c_{+}+c_{-}\right)^{2}} i f^{a}{ }_{c b} t^{b}+\mathcal{O}\left(f^{4}\right) \\
B^{a}{ }_{c} & =\frac{c_{+}}{\left(c_{+}+c_{-}\right)^{2}} i f^{a}{ }_{c b} t^{b}+\mathcal{O}\left(f^{4}\right) .
\end{aligned}
$$

We note that one can reach the same conclusion by computing the OPE between a current and both sides of the equation (4.10), i.e. by demanding compatibility with the proportionality relation between the operators $\partial \phi$ and $t_{a}: j_{L, z}^{a} \phi:$.

\section{E.3 Stress-tensor-primary OPE at order $f^{2}$}

Here we present the computation of the OPE between the stress-energy tensor and a primary field $\phi$. This computation relies on the prescription of appendix A, and on the current-current and current-primary OPEs (2.5) and (4.2). Since we computed these OPEs up to order $f^{2}$, we will also obtain the stress-tensor OPE up to order $f^{2}$.

$$
\begin{aligned}
\phi(z) 2 c_{1} T(w)= & \lim _{: x \rightarrow w:} \phi(z) j_{L, z}^{a}(x) j_{L, z}^{b}(w) \kappa_{b a} \\
= & \kappa_{a b} \lim _{: x \rightarrow w:}\left[\left(-\frac{c_{+}}{c_{+}+c_{-}} \frac{t^{a} \phi(z)}{x-z}+: j_{L, z}^{a} \phi:(x)\right.\right. \\
& \left.+A^{a}{ }_{c} \log |z-x|^{2}: j_{L, z}^{c} \phi:(x)+B^{a}{ }_{c} \frac{\bar{z}-\bar{x}}{z-x}: j_{L, \bar{z}}^{c} \phi:(x)+\ldots\right) j_{L, z}^{b}(w) \\
& \left.+j_{L, z}^{a}(x)\left(-\frac{c_{+}}{c_{+}+c_{-}} \frac{t^{b} \phi(w)}{w-z}+\mathcal{O}\left((z-w)^{0}\right)\right)\right]
\end{aligned}
$$


Let us first consider the first term in the previous expression. According to the prescription given in appendix $\mathrm{A}$, we have to evaluate the operator $\phi(z)$ at the point $x$ before we take the OPE with the remaining current $j_{L, z}^{b}(w)$. So we rewrite this term as:

$$
\begin{aligned}
& \kappa_{a b} \lim _{: x \rightarrow w:}\left(-\frac{c_{+}}{c_{+}+c_{-}} \frac{t^{a}}{x-z} \sum_{n, \bar{n}=0}^{\infty} \frac{(z-x)^{n}}{n !} \frac{(\bar{z}-\bar{x})^{\bar{n}}}{\bar{n} !} \partial^{n} \bar{\partial}^{\bar{n}} \phi(x)\right) j_{L, z}^{b}(w) \\
&=\kappa_{a b} t^{a} \frac{c_{+}}{c_{+}+c_{-}} \lim _{x \rightarrow w:} \sum_{n, \bar{n}=0}^{\infty} \frac{(z-x)^{n-1}}{n !} \frac{(\bar{z}-\bar{x})^{\bar{n}}}{\bar{n} !} \partial_{x}^{n} \bar{\partial}_{x}^{\bar{n}}\left(-\frac{c_{+}}{c_{+}+c_{-}} \frac{t^{b} \phi(x)}{w-x}-: j_{L, z}^{b} \phi:(w)+\ldots\right) \\
&=-\kappa_{a b} t^{a} t^{b}\left(\frac{c_{+}}{c_{+}+c_{-}}\right)^{2} \lim _{: x \rightarrow w:} \sum_{n, \bar{n}=0}^{\infty} \frac{(z-x)^{n-1}}{n !} \frac{(\bar{z}-\bar{x})^{\bar{n}}}{\bar{n} !} \partial_{x}^{n} \bar{\partial}_{x}^{\bar{n}} \\
&\left(\frac{1}{w-x} \sum_{m, \bar{m}=0}^{\infty} \frac{(x-w)^{m}}{m !} \frac{(\bar{x}-\bar{w})^{\bar{m}}}{\bar{m} !} \partial^{m} \bar{\partial}^{\bar{m}} \phi(w)\right)-\kappa_{a b} t^{a} \frac{c_{+}}{c_{+}+c_{-}} \frac{: j_{L, z}^{b} \phi:(w)}{w-z}+\ldots
\end{aligned}
$$

In the previous lines we only kept track of the operators that will lead to poles in the final result. We evaluated the operator $\phi$ at the point $w$ so that the action of the derivatives is easier to take care of:

$$
\begin{aligned}
=-\kappa_{a b} t^{a} t^{b}\left(\frac{c_{+}}{c_{+}+c_{-}}\right)^{2} \lim _{: x \rightarrow w:} \sum_{n, \bar{n}=0}^{\infty} \frac{(z-x)^{n-1}}{n !} \frac{(\bar{z}-\bar{x})^{\bar{n}}}{\bar{n} !} \\
\quad\left(\sum_{m, \bar{m}=0}^{\infty}(-1) \frac{(x-w)^{m-n-1}}{m(m-n-1) !} \frac{(\bar{x}-\bar{w})^{\bar{m}-\bar{n}}}{(\bar{m}-\bar{n}) !} \partial^{m} \bar{\partial}^{\bar{m}} \phi(w)\right)-\kappa_{a b} t^{a} \frac{c_{+}}{c_{+}+c_{-}} \frac{: j_{L, z}^{b} \phi:(w)}{w-z}+\ldots
\end{aligned}
$$

The regular limit gives a non-zero result for the anti-holomorphic factor only if $\bar{m}-\bar{n}=0$. For the holomorphic factor, one needs $m-n-1=0$. Notice that the terms with $n=m=0$ also contributes with a non-vanishing term. Eventually we obtain:

$$
\begin{aligned}
& \kappa_{a b} t^{a} t^{b}\left(\frac{c_{+}}{c_{+}+c_{-}}\right)^{2}\left(\sum_{n, \bar{n}=0}^{\infty} \frac{(z-w)^{n-1}}{(n+1) !} \frac{(\bar{z}-\bar{x})^{\bar{n}}}{\bar{n} !} \partial^{n+1} \bar{\partial}^{\bar{n}} \phi(w)\right. \\
&\left.+\sum_{\bar{n}=0}^{\infty} \frac{1}{(z-w)^{2}} \frac{(\bar{z}-\bar{x})^{\bar{n}}}{\bar{n} !} \bar{\partial}^{\bar{n}} \phi(w)\right)-\kappa_{a b} t^{a} \frac{c_{+}}{c_{+}+c_{-}} \frac{: j_{L, z}^{b} \phi:(w)}{w-z}+\ldots \\
&=\kappa_{a b} t^{a} t^{b}\left(\frac{c_{+}}{c_{+}+c_{-}}\right)^{2} \frac{\phi(z)}{(z-w)^{2}}-\kappa_{a b} t^{a} \frac{c_{+}}{c_{+}+c_{-}} \frac{: j_{L, z}^{b} \phi:(w)}{w-z}+\ldots
\end{aligned}
$$

This completes the evaluation of the first term in (E.11). The other terms are much easier to deal with. The only non-trivial part is the computation the OPE between a current $j_{L, z}^{b}(w)$ and the composite operators : $j_{L, z}^{c} \phi:(z)$ and $: j_{L, \bar{z}}^{c} \phi:(z)$. Since the coefficients $A^{a}{ }_{c}$ and $B^{a}{ }_{c}$ already are of order $f^{2}$, we only need to know these OPEs at order $f^{0}$. We find:

$$
\begin{gathered}
j_{L, z}^{b}(w): j_{L, z}^{c} \phi:(z)=\left(c_{1} \kappa^{b c}+\frac{c_{-}\left(c_{2}-g\right)-c_{+} c_{2}}{c_{+}+c_{-}} f^{b c}{ }_{d} t^{d}\right) \frac{\phi(z)}{(w-z)^{2}} \\
-\frac{c_{+}}{c_{+}+c_{-}} \frac{t^{b}: j_{L, z}^{c} \phi:(z)}{w-z}+\mathcal{O}\left(f^{2}\right)
\end{gathered}
$$




$$
\begin{gathered}
j_{L, z}^{b}(w): j_{L, \bar{z}}^{c} \phi:(z)=\left(\tilde{c} \kappa^{b c}+\frac{c_{-}\left(c_{2}-g\right)+c_{+}\left(c_{4}-g\right)}{c_{+}+c_{-}} f^{b c} d^{t^{d}}\right) \phi(z) 2 \pi \delta^{(2)}(w-z) \\
-\frac{c_{+}}{c_{+}+c_{-}} \frac{t^{b}: j_{L, \bar{z}}^{c} \phi:(z)}{w-z}+\mathcal{O}\left(f^{2}\right) .
\end{gathered}
$$

All the terms that appear in these OPEs give zero once contracted either with $A^{a}{ }_{c} \kappa_{a b}$ or with $B^{a}{ }_{c} \kappa_{a b}$. In particular factors of the form $f^{a}{ }_{b c} c^{c} t^{b}$ vanish since the dual Coxeter number is zero. Gathering everything we obtain:

$$
\begin{aligned}
\phi(z) 2 c_{1} T(w)= & \frac{c_{+}^{2}}{\left(c_{+}+c_{-}\right)^{2}} t^{a} t^{b} \kappa_{a b} \frac{\phi(z)}{(z-w)^{2}}-\frac{2 c_{+}}{c_{+}+c_{-}} \frac{\kappa_{a b} t^{a}: j_{L, z}^{b} \phi:(z)}{w-z} \\
& +\mathcal{O}(z-w)^{0}+\mathcal{O}\left(f^{2}\right) .
\end{aligned}
$$

The previous result is true only up to terms of order $f^{2}$, since a term of order $f^{4}$ in the current-primary OPE may give a term of order $f^{2}$ once contracted with an additional current (see lemma (C.11)). We rewrite the result as:

$$
T(w) \phi(z)=\frac{f^{2}}{2} \frac{t^{a} t^{b} \kappa_{a b} \phi(z)}{(z-w)^{2}}+\frac{1}{c_{+}} \frac{\kappa_{a b} t^{a}: j_{L, z}^{b} \phi:(z)}{w-z}+\mathcal{O}(z-w)^{0}+\mathcal{O}\left(f^{4}\right)
$$

This concludes the proof of equation (4.6) in section 4 .

\section{F The mode expansion on the cylinder}

When the theory is defined on a cylinder we can expand the operators in modes by means of a Fourier transform along the compact coordinate. Then we can convert the currentcurrent OPEs into graded commutation relations for the modes of the currents. This was done for the current algebra (2.5) in [4]. In this appendix we give the translation of the left current - right current OPEs (3.15), (3.16) in terms of commutation relations. We use the same techniques as in section 5 of [4]. To simplify the notation we do not write explicitly the subscript $L$ or $R$ on the currents since it is redundant with the different notation for the left and right adjoint representations. We expand the currents and the adjoint operator in modes:

$$
\begin{aligned}
j_{z}^{a}(\sigma, \tau) & =+i \sum_{n \in Z} e^{-i n \sigma} j_{z, n}^{a}(\tau) \\
j_{\bar{z}}^{a}(\sigma, \tau) & =-i \sum_{n \in Z} e^{-i n \sigma} j_{\bar{z}, n}^{a}(\tau) \\
j_{z}^{\bar{a}}(\sigma, \tau) & =+i \sum_{n \in Z} e^{-i n \sigma} j_{z, n}^{\bar{a}}(\tau) \\
j_{\bar{z}}^{\bar{a}}(\sigma, \tau) & =-i \sum_{n \in Z} e^{-i n \sigma} j_{\bar{z}, n}^{\bar{a}}(\tau) \\
\mathcal{A}^{a \bar{a}}(\sigma, \tau) & =\sum_{n \in Z} e^{-i n \sigma} \mathcal{A}^{a \bar{a}}(\tau) .
\end{aligned}
$$

We obtain the commutation relations:

$$
\left[j_{z, n}^{a}, j_{z, m}^{\bar{a}}\right]=+\frac{c_{+} c_{-}}{c_{+}+c_{-}} \frac{c_{-} m-c_{+} n}{c_{+}+c_{-}} \mathcal{A}_{n+m}^{a \bar{a}}
$$




$$
\begin{aligned}
& {\left[j_{\bar{z}, n}^{a}, j_{\bar{z}, m}^{\bar{a}}\right]=-\frac{c_{+} c_{-}}{c_{+}+c_{-}} \frac{c_{+} m-c_{-} n}{2} \mathcal{A}_{n+m}^{a \bar{a}}} \\
& {\left[j_{z, n}^{a}, j_{\bar{z}, m}^{\bar{a}}\right]=-\frac{c_{+}^{2} c_{-}}{\left(c_{+}+c_{-}\right)^{2}}(m+n) \mathcal{A}_{n+m}^{a \bar{a}}} \\
& {\left[j_{\bar{z}, n}^{a}, j_{z, m}^{\bar{a}}\right]=+\frac{c_{+} c_{-}^{2}}{\left(c_{+}+c_{-}\right)^{2}}(m+n) \mathcal{A}_{n+m}^{a \bar{a}},}
\end{aligned}
$$

as well as the standard commutation relations between the modes of the currents and the left-right adjoint primary (as determined by their OPE), and the left-left commutation relations calculated in [4].

In [4] it was shown that the combination of left current components $j_{z, n}^{a}-j_{\bar{z}, n}^{a}$ generate a Kac-Moody algebra at integer level $k$. This is also the case for the right combination $j_{z, m}^{\bar{a}}-j_{\bar{z}, m}^{\bar{a}}$. As a consequence of the above commutation relations, we find moreover that the left and right Kac-Moody subalgebras commute:

$$
\left[j_{z, n}^{a}-j_{\bar{z}, n}^{a}, j_{z, m}^{\bar{a}}-j_{\bar{z}, m}^{\bar{a}}\right]=0 .
$$

Only the zero modes of these affine currents commute with the worldsheet Hamiltonian.

\section{G Classical integrability}

In this appendix, we will show that principal chiral models with or without Wess-Zumino term are classically integrable. We generalize here the standard calculation to the case with non-zero Wess-Zumino term. The equations of motion $d * j=0$ for the model written in terms of the left current components read:

$$
\bar{\partial} j_{z}^{a}+\partial j_{\bar{z}}^{a}=0
$$

where we have that:

$$
\begin{aligned}
& j_{z}=-\frac{1}{2}\left(\frac{1}{f^{2}}+k\right) \partial g g^{-1} \\
& j_{\bar{z}}=-\frac{1}{2}\left(\frac{1}{f^{2}}-k\right) \bar{\partial} g g^{-1} .
\end{aligned}
$$

As before, the coefficient of the principal chiral model term is $1 / f^{2}$ and the Wess-Zumino term has coefficient $k$. The Maurer-Cartan equation $d\left(d g g^{-1}\right)=d g g^{-1} \wedge d g g^{-1}$ is:

$$
-\frac{1}{2}\left(\frac{1}{f^{2}}-k\right) \bar{\partial} j_{z}^{a}+\frac{1}{2}\left(\frac{1}{f^{2}}+k\right) \partial j_{\bar{z}}^{a}-i f_{b c}^{a} j_{z}^{c} j_{\bar{z}}^{b}=0 .
$$

In this context it is easier to work with the canonical right invariant one-form:

$$
\omega=d g g^{-1}
$$

and rewrite the equations of motion in terms of $\omega$ and the coefficients $c_{ \pm}$defined as in the bulk of the paper:

$$
\begin{aligned}
\bar{\partial} \omega_{z} & =\frac{c_{-}}{c_{+}+c_{-}}\left[\omega_{z}, \omega_{\bar{z}}\right] \\
\partial \omega_{\bar{z}} & =-\frac{c_{+}}{c_{+}+c_{-}}\left[\omega_{z}, \omega_{\bar{z}}\right] .
\end{aligned}
$$


Now consider a connection which is a function of a spectral parameter $\lambda$ :

$$
A(\lambda)=\frac{2}{1+\lambda} \frac{c_{+}}{c_{+}+c_{-}} \omega_{z} d z+\frac{2}{1-\lambda} \frac{c_{-}}{c_{+}+c_{-}} \omega_{\bar{z}} d \bar{z}
$$

and compute the curvature of the connection:

$$
F_{\bar{z} z}=\frac{2}{1+\lambda} \frac{c_{+}}{c_{+}+c_{-}} \bar{\partial} \omega_{z}-\frac{2}{1-\lambda} \frac{c_{-}}{c_{+}+c_{-}} \partial \omega_{\bar{z}}-\frac{c_{+}}{c_{+}+c_{-}} \frac{c_{-}}{c_{+}+c_{-}} 2\left(\frac{1}{1+\lambda}+\frac{1}{1-\lambda}\right)\left[\omega_{z}, \omega_{\bar{z}}\right] .
$$

Flatness of the connection for all values of the spectral parameter $\lambda$ is equivalent to the validity of the equations of motion (G.5). Using the on-shell flat connection, we can define an infinite set of conserved charges, for instance by calculating the traced holonomy for the model on a circle times time, and expanding in the spectral parameter. The infinite set of conserved charges renders the theory classically integrable. The theory can then be studied using the powerful tools of integrability.

Open Access. This article is distributed under the terms of the Creative Commons Attribution Noncommercial License which permits any noncommercial use, distribution, and reproduction in any medium, provided the original author(s) and source are credited.

\section{References}

[1] N. Berkovits, C. Vafa and E. Witten, Conformal field theory of AdS background with Ramond-Ramond flux, JHEP 03 (1999) 018 [hep-th/9902098] [SPIRES].

[2] M. Bershadsky, S. Zhukov and A. Vaintrob, $P S L(n-n) \sigma$-model as a conformal field theory, Nucl. Phys. B 559 (1999) 205 [hep-th/9902180] [SPIRES].

[3] A. Babichenko, Conformal invariance and quantum integrability of $\sigma$-models on symmetric superspaces, Phys. Lett. B 648 (2007) 254 [hep-th/0611214] [SPIRES].

[4] S.K. Ashok, R. Benichou and J. Troost, Conformal current algebra in two dimensions, JHEP 06 (2009) 017 [arXiv:0903.4277] [SPIRES].

[5] L. Rozansky and H. Saleur, Quantum field theory for the multivariable Alexander-Conway polynomial, Nucl. Phys. B 376 (1992) 461 [SPIRES].

[6] V. Schomerus and H. Saleur, The GL(1-1) WZW model: from supergeometry to logarithmic CFT, Nucl. Phys. B 734 (2006) 221 [hep-th/0510032] [SPIRES].

[7] G. Götz, T. Quella and V. Schomerus, The WZNW model on PSU $(1,1 \mid 2)$, JHEP 03 (2007) 003 [hep-th/0610070] [SPIRES].

[8] N. Read and H. Saleur, Exact spectra of conformal supersymmetric nonlinear $\sigma$-models in two dimensions, Nucl. Phys. B 613 (2001) 409 [hep-th/0106124] [SPIRES].

[9] T. Quella, V. Schomerus and T. Creutzig, Boundary spectra in superspace $\sigma$-models, JHEP 10 (2008) 024 [arXiv:0712.3549] [SPIRES].

[10] V. Mitev, T. Quella and V. Schomerus, Principal chiral model on superspheres, JHEP 11 (2008) 086 [arXiv:0809.1046] [SPIRES].

[11] C. Candu, V. Mitev, T. Quella, H. Saleur and V. Schomerus, The $\sigma$-model on complex projective superspaces, JHEP 02 (2010) 015 [arXiv:0908.0878] [SPIRES]. 
[12] C. Candu, T. Creutzig, V. Mitev and V. Schomerus, Cohomological reduction of $\sigma$-models, arXiv: 1001.1344 [SPIRES].

[13] J.M. Maldacena, The large- $N$ limit of superconformal field theories and supergravity, Adv. Theor. Math. Phys. 2 (1998) 231 [Int. J. Theor. Phys. 38 (1999) 1113] [hep-th/9711200] [SPIRES].

[14] N. Gromov, V. Kazakov and P. Vieira, Exact spectrum of anomalous dimensions of planar $N=4$ supersymmetric Yang-Mills theory, Phys. Rev. Lett. 103 (2009) 131601 [arXiv: 0901.3753] [SPIRES].

[15] S.K. Ashok, R. Benichou and J. Troost, Asymptotic symmetries of string theory on $A d S_{3} \times S^{3}$ with Ramond-Ramond fluxes, JHEP 10 (2009) 051 [arXiv:0907.1242] [SPIRES].

[16] V.G. Knizhnik and A.B. Zamolodchikov, Current algebra and Wess-Zumino model in two dimensions, Nucl. Phys. B 247 (1984) 83 [SPIRES].

[17] P. Di Francesco, P. Mathieu and D. Senechal, Conformal field theory, Springer, New York, U.S.A. (1997).

[18] M.R. Gaberdiel, An algebraic approach to logarithmic conformal field theory, Int. J. Mod. Phys. A 18 (2003) 4593 [hep-th/0111260] [SPIRES].

[19] M. Flohr, Bits and pieces in logarithmic conformal field theory, Int. J. Mod. Phys. A 18 (2003) 4497 [hep-th/0111228] [SPIRES].

[20] M. Lüscher, Quantum nonlocal charges and absence of particle production in the two-dimensional nonlinear $\sigma$-model, Nucl. Phys. B 135 (1978) 1 [SPIRES]; 